\title{
The gaseous environments of quasars: associate absorption lines with density and distance constraints
}

\author{
Chen Chen, ${ }^{1,2 \star}$ Fred Hamann, ${ }^{2}$ Leah Simon ${ }^{1,3}$ and Thomas Barlow ${ }^{4}$ \\ ${ }^{1}$ Department of Astronomy, University of Florida, 211 Bryant Space Science Center, FL 32611, USA \\ ${ }^{2}$ Department of Physics \& Astronomy, University of California, Riverside, CA 92521, USA \\ ${ }^{3}$ Hands On Labs, 750 W. Hampden Ave., Suite 100, Englewood, CO 80110, USA \\ ${ }^{4}$ Caltech Optical Observatories, 1200 E California Blvd, Pasadena, CA 91125, USA
}

Accepted 2018 September 13. Received 2018 September 12; in original form 2018 April 20

\begin{abstract}
Associated absorption lines (AALs) in quasar spectra are valuable probes of the gas kinematics and physical conditions in quasar environments. The host galaxies are by definition in an active evolution stage that might involve large-scale blowouts and/or cold-mode accretion (infall) from the intergalactic medium (IGM). We discuss rest-frame UV spectra of four redshift 2-3 quasars selected to have low-ionization AALs of Si II or C II that place unique density and distance constraints on the absorbers. Our analysis of the AALs yields the following results. One of the quasars, Q0119-046, has a rich complex of 11 AAL systems that appear to be infalling at measured speeds from $\sim 0$ to $\sim 1150 \mathrm{~s}^{-1}$ at distance $\sim 5.7 \mathrm{kpc}$ from the quasar. The range of ions detected, up to Ne VIII, indicates a wide range of densities from $\sim 4$ to $\sim 2500 \mathrm{~cm}^{-3}$. Partial covering the quasar emission source requires cloud sizes $<1 \mathrm{pc}$ and possibly $<0.01 \mathrm{pc}$. The short dissipation times of these small clouds suggest that they are created in situ at their observed location, perhaps as dense condensations in cold-mode accreting gas from IGM. The AALs in the other three quasars have outflow speeds from $\sim 1900$ to $\sim 3000 \mathrm{~s}^{-1}$. Some of them also appear to have a range of densities based on the range of ions detected, including $\mathrm{Mg}$ I $\lambda 2853$ in Q0105+061. However, the absence of excited-state AALs yields only upper limits on their gas densities $\left(\lesssim 150 \mathrm{~cm}^{-3}\right)$ and large minimum distances $(\gtrsim 40 \mathrm{kpc}$ ) from the quasars. These AALs might represent highly extended quasar-driven outflows, although their actual physical relationships to the quasars cannot be established.
\end{abstract}

Key words: galaxies: evolution-quasars: absorption lines-quasars: general.

\section{INTRODUCTION}

High-redshift quasars in the centres of massive galaxies signal an active early stage of massive galaxy evolution. Popular evolution models suggest that the black hole accretion that defines quasar activity is accompanied by a rapid burst of star formation in the host galaxies, triggered perhaps by a galaxy merger or interaction (Sanders et al. 1988; Elvis 2006; Hopkins et al. 2008; Veilleux et al. 2009; Hopkins et al. 2016). This activity is expected to continue until powerful outflows (feedback) driven by the central quasar and/or star formation lead to a blowout of gas and dust that quenches the starburst and cuts off the fuel supply for black hole accretion (Silk \& Rees 1998; Kauffmann \& Haehnelt 2000; King 2003; Scannapieco \& Oh 2004; Di Matteo, Springel \& Hernquist 2005; Ostriker et al. 2010; Debuhr, Quataert \& Ma 2012; Rupke \& Veilleux 2013; Cicone et al. 2014; Rupke, Gültekin \& Veilleux 2017)
Infalling gas from the intergalactic medium (IGM, e.g. cold mode accretion) is also expected to be important during the early evolution stages to build galaxy mass, trigger star formation, and fuel the central black holes (Katz et al. 2003; Kereš et al. 2009, 2012). Recent observations show that massive gas reservoirs are indeed present around high-redshift quasars, and that they are more extended and more massive around quasar hosts than similar inactive (non-quasar) galaxies (e.g. Prochaska, Lau \& Hennawi 2014; Johnson, Chen \& Mulchaey 2015; Martin et al. 2015, 2016; Borisova et al. 2016; Bouché et al. 2016; Ho et al. 2017). These gas reservoirs are consistent with enhanced infall/cold-mode accretion from the IGM during the early/active quasars stages of massive galaxy evolution. It is likely that infall and outflow occur together if coldmode accretion is involved in triggering the starbursts and quasars that also drive feedback (Costa, Sijacki \& Haehnelt 2014; Nelson et al. 2015; Suresh et al. 2015).

Associated absorption lines (AALs) in quasar spectra are unique tools to study the gaseous environments of quasars and test models of massive galaxy evolution. AALs have narrow velocity widths (less than a few hundred $\mathrm{km} \mathrm{s}^{-1}$ ) much different from broad 
Table 1. Quasar data including the name, emission-line redshift, telescope-instrument used for observations, observation date (dd/mm/year), rest wavelength ranges, and spectral resolution $R=\lambda / \Delta \lambda$.

\begin{tabular}{lccccc}
\hline Quasar & $z_{\mathrm{em}}$ & Instrument & Obs. date & $\lambda_{\text {rest }}(\AA)$ & $R$ \\
\hline Q0105+061 & $1.960^{a}$ & VLT-UVES & $09 / 22 / 2003$ & $1184-1604$ & 80000 \\
& & & $2044-3352$ & 110000 \\
Q0119-046 & $1.9635^{b}$ & Keck-HIRES & $03 / 12 / 1996$ & $1147-1989$ & 45000 \\
& & HST-FOS & $09 / 24 / 1996$ & $750-1106$ & 1300 \\
Q0334-204 & $3.132^{c}$ & VLT-UVES & $09 / 23 / 2003$ & $848-1077$ & 80000 \\
& & & & $1152-1654$ & 110000 \\
Q2044-168 & $1.939^{d}$ & VLT-UVES & $09 / 22 / 2003$ & $1193-1606$ & 80000 \\
& & & & $2059-3376$ & 110000 \\
\hline
\end{tabular}

${ }^{a}$ Ulrich (1989).

${ }^{b}$ Steidel \& Sargent (1991).

${ }^{c}$ Tytler \& Fan (1992).

${ }^{d}$ Tytler et al. (2004).

absorption lines (BALs), whose velocity widths $\gtrsim 2000 \mathrm{~s}^{-1}$ clearly identify high-speed quasar-driven outflows (Anderson et al. 1987; Weymann et al. 1991; Hamann \& Sabra 2004; Simon \& Hamann 2010; Muzahid et al. 2013). The term 'associated' means they have absorption redshifts within several thousand $\mathrm{km} \mathrm{s}^{-1}$ of the quasar emission-line redshift (i.e. $z_{\mathrm{abs}} \approx z_{\mathrm{em}}$, Weymann et al. 1979 ; Foltz et al. 1986; Hamann 1997). It is known from statistical studies of large quasar samples that most AALs are intrinsic to the quasars, i.e. they form broadly within the environment of the quasar or its host galaxy (Nestor, Hamann \& Rodriguez Hidalgo 2008; Wild et al. 2008; Perrotta et al. 2016). However, AALs can have a wide range of physical origins, from outflows near the quasars, to extended haloes in the host galaxies, to cosmologically intervening gas or galaxies unrelated to the quasars (Sargent, Boksenberg \& Young 1982; Tripp, Lu \& Savage 1996; Hamann et al. 2001; D’Odorico et al. 2004; Hamann et al. 2011).

Several observational tests have been proposed to determine if individual AAL systems are likely intrinsic (Barlow, Hamann \& Sargent 1997; Hamann, Barlow \& Junkkarinen 1997a; Ganguly et al. 1999; Hamann \& Ferland 1999; Narayanan et al. 2004; Wise et al. 2004; Misawa et al. 2007b). They include (1) high gas densities inferred from excited-state absorption lines (that require a close proximity to the quasars based on photoionization constraints), (2) absorption line variability, (3) partial covering of the background light source that, for quasars, requires very small absorbing clouds, and (4) line profiles indicative of gas flows because they are too broad and smooth compared to thermal velocities ( see also Ganguly et al. 1999; Hamann \& Ferland 1999; Srianand \& Petitjean 2000; Schaye, Carswell \& Kim 2007; Arav et al. 2008). These properties tend to go together. They are indicative of an intrinsic origin because they are more readily understood in terms of the dense dynamic of quasars compared to the larger, more quiescent, and lower density clouds expected for intervening absorption (Rauch 1998).

In this paper, we describe a study to understand the nature and origins of low-density associated absorbers that might reside in the extended host galaxies of quasars. This is an interesting subset of AALs because the extended galactic environments are where we might find evidence for cold mode accretion or quasar-driven winds directly interacting with the galactic interstellar medium. We describe the quasar sample and the data used in Section 2. We fit the AALs to measure the kinematics, column densities, and covering fractions in Section 3. We analyse the physical properties including ionization, electron density, metallicity, and radial distance in
Section 4, and in Section 5 we discuss the origins and dynamics of absorption-line clouds. We conclude with a summary in Section 6. Throughout this paper, we adopt a cosmology with $H_{0}=71 \mathrm{~s}^{-1}$ $\mathrm{Mpc}^{-1}, \Omega_{\mathrm{M}}=0.27$, and $\Omega_{\Lambda}=0.73$.

\section{DATA OVERVIEW}

\subsection{Quasar sample}

We select four AAL quasars for our study from samples observed previously by our team using the High Resolution Echelle Spectrometer (HIRES) at the W. M. Keck Observatory (Keck) or the Utlraviolet and Visual Echelle Spectrometer (UVES) at the ESO Very Large Telescope (VLT). We require that the quasars have low-ionization AALs of C II or Si II detected in these existing highresolution spectra. This sample is not intended to be representative of all quasar AALs. In particular, the low-ionization $\mathrm{C}$ II and $\mathrm{Si}$ II lines are rare in AAL systems. However, these lines place unique constraints on the absorber densities and locations. They can, therefore, provide unique insights in the nature of the gas flows around quasars and their extended host galaxies.

The quasars, their redshifts, and some basic information about the data are listed in Table 1. Q0105+061, Q0334-204, and Q2044-168 are the only quasars with detected C II or Si II AALs in the larger sample of 24 AAL quasars described by Simon, Hamann \& Pettini (2012). The 24 AAL quasars were selected from the Sloan Digital Sky Survey (SDSS, York et al. 2000) to be bright (apparent magnitude $\lesssim 19$ ) with CIV AALs at redshifts $\gtrsim 2$. Q0119-046 is selected from unpublished high-resolution spectra obtained with both Keck and the Hubble Space Telescope (HST). Q0119-046 and Q2044-168 are radio-loud (Murphy et al. 2010; Chhetri et al. 2013), while Q0105+061 and Q0334-204 are radioquiet (Sramek \& Weedman 1980; Robson et al. 1985). The AALs in Q0119-046 were studied previously by Sargent et al. (1982). Here we present data with higher spectral resolution and higher signal-tonoise ratios, with wider wavelength coverage in the $H S T$ data that reveals additional absorption lines including Ne VIII $\lambda \lambda 770,780$, O VI $\lambda \lambda 1032,1038$, and the Lyman lines to yield important new results on the nature of its AAL absorbers.

Accurate systemic redshifts are important to judge infall versus outflow for the AALs and estimate the outflow kinetic energies. Redshifts derived from UV broad emission lines are known to be uncertain because the lines can be shifted in the quasar frame, with 
different lines shifted by different amounts. High-ionization lines like C IV $\lambda \lambda 1548,1551$ are typically blueshifted by several hundred $\mathrm{km} \mathrm{s}^{-1}$ compared to narrow forbidden lines such as [O III] $\lambda 5007$, which is generally regarded to be the best UV/optical indicator of quasar systemic redshifts (Gaskell 1982; Shen et al. 2007; Wang et al. 2011). Low-ionization permitted lines such as $\mathrm{Mg}$ II $\lambda 2800$ can also be good redshift indicators because their shifts relative to [O III] are typically $\sim 100 \mathrm{~s}^{-1}$ (Richards et al. 2002). We search the literature to find the best available redshift for each quasar based on the lines measured and the data quality. The results are listed in Table 1. Below are some specific notes.

Q0105+061: Ulrich (1989) performed Gaussian fit (twocomponent fits for double lines) to measure the emission lines C IV, C III] $\lambda 1909$, and Si IV+O IV] $\lambda 1398$ in the spectrum with resolution $7 \AA$. The redshifts are $1.955,1.953,1.965$, respectively, from the measurements of the three emission lines. We adopt $z_{\mathrm{em}}=$ $1.960 \pm 0.005\left( \pm 507 \mathrm{~s}^{-1}\right)$ by weighting their individual line measurements based on the emission line widths.

Q0119-046: Steidel \& Sargent (1991) measured Mg II to determine the emission-line redshift $z_{\mathrm{em}}=1.9635$ by calculating the flux-weighted mean wavelength of the line above 85 percent of its peak height. Given the spectral resolution and noise in the data, they estimated that the errors on their line positions are typically $\sim 50-100 \mathrm{~s}^{-1}$. We adopt the value $1.9635 \pm 0.0020\left( \pm 202 \mathrm{~s}^{-1}\right)$, with a generous uncertainty to include the measurement uncertainty and the typical offset, $\sim 100 \mathrm{~s}^{-1}$, of $\mathrm{Mg}$ II relative to [O III] emission lines (Richards et al. 2002; Shen et al. 2007).

Q0334-204: Tytler \& Fan (1992) presented a new maximumlikelihood method, which gave more accurate results than previous studies, to measure emission lines of Ly $\alpha \lambda 1216$, Si IV+O IV], C IV, and C III]. They obtained a value of $3.1322 \pm 0.0013\left( \pm 94 \mathrm{~s}^{-1}\right)$. We directly use this redshift.

Q2044-168: Tytler et al. (2004) measured the emission-line redshifts using lines Ly $\alpha, \mathrm{N}$ V $\lambda 1240$, Si IV+O IV], C IV, C III] with a typical error of $5 \AA$ for the peak wavelengths, and the redshifts determined by these lines are 1.939, 1.939, 1.936, 1.939, 1.938, respectively. We adopt the value of $1.939 \pm 0.001\left( \pm 102 \mathrm{~s}^{-1}\right)$ by weighting their individual line measurements.

\subsection{Observations and data reduction}

Basic information about the observations is listed in Table 1. Spectra of Q0119-046 were observed with High Resolution Echelle Spectrometer (HIRES) on Keck and Faint Object Spectrograph (FOS) on the HST. The other three quasars were observed with UV-Visual Echelle Spectrograph (UVES) on the VLT. All of the spectra cover important absorption lines from at least Ly $\alpha \lambda 1216$ to C IV $\lambda \lambda 1548,1551$ in the quasar rest frame. The HST-FOS spectrum of Q0119-046 provides additional UV coverage below the Lyman limit. The wavelength scales throughout this paper are vacuum heliocentric. We used standard techniques to reduce the KeckHIRES and VLT-UVES spectra. In particular, for the Keck-HIRES spectra, we used a software package MAKEE (as described in Barlow \& Sargent 1997; Hamann et al. 1997b) for initial data reduction and spectral extraction. These procedures are described in detail in Hamann et al. (2001). The VLT-UVES data reduction procedures are described in Simon (2011).

We use the IRAF ${ }^{1}$ software package for additional data processing. In particular, we normalize the reduced Keck and VLT spectra

${ }^{1}$ Image Reduction and Analysis Facility (IRAF) is maintained and distributed by the National Optical Astronomy Observatories.

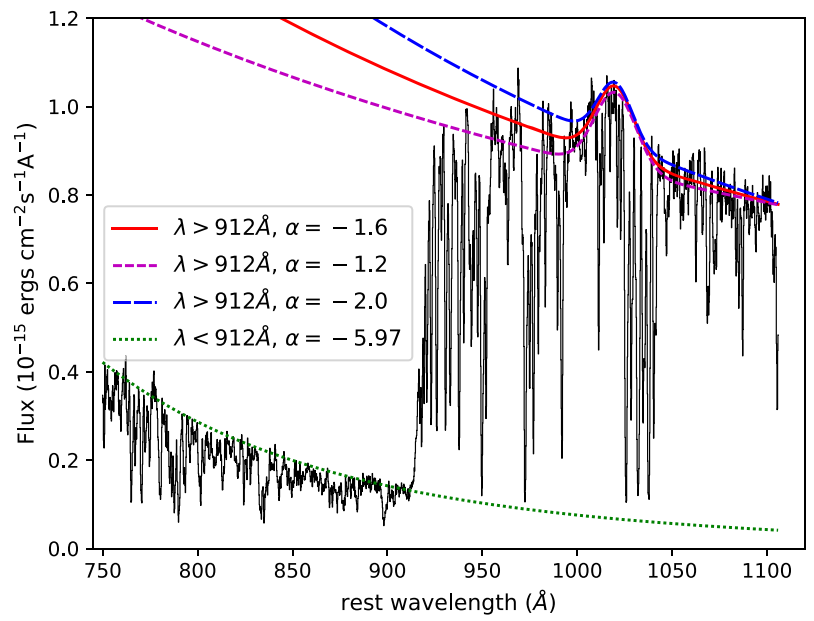

Figure 1. HST spectrum of Q0119-046. The dotted green line shows the fit of the continuum at $\lambda<912 \AA$, and the solid red line shows the best estimation of the continuum at $\lambda>912 \AA$, including O VI emission line. The dashed blue and mauve lines show upper and lower limits at $\lambda>912$ Å, respectively.

to unity by fitting a pseudo-continuum to each quasar spectrum, including the emission lines. For regions with few absorption lines, we apply a polynomial fit locally around the absorption lines we will measure for our study. For the regions where the continuum is affected by significant absorption, e.g. in the Ly $\alpha$ forest, we visually inspect the spectra to find small segments of continuum not affected by absorption or obvious noise spikes. We then interpolate between these segments, fitting the entire region with a low-order polynomial.

\subsubsection{HST-FOS spectrum of Q0119-046}

For Q0119-046 only, we include the UV spectrum obtained with HST-FOS shown in Fig. 1. We retrieved this spectrum already reduced and calibrated from the Mikulski Archive for Space Telescopes. We attempted to test the wavelength calibrations using the Galactic absorption lines of $\mathrm{Mg}$ II $\lambda \lambda 2796,2804$ and Fe II $\lambda \lambda 2587$, 2600 (Schneider et al. 1993). However, these lines are contaminated in the Ly $\alpha$ forest and not useful. Instead, we compare the Ly $\alpha$ AALs measured in the Keck spectrum (labeled as 2 and 7 in Fig. 3) to the corresponding Ly $\beta$, Ly $\gamma$, and Ly $\delta$ AALs in the HST spectrum (labelled as $2^{*}$ and $7^{*}$ in Fig. 3). Our fits to these lines (described below) show no significant differences in the centroid redshifts and, therefore, we do not apply any corrections to the HST spectrum wavelengths.

The HST spectrum has a much lower resolution than the Keck spectrum, but it covers shorter wavelengths with a variety of important AALs, including Ne VIII $\lambda \lambda 770,780$, O VI $\lambda \lambda 1032$, 1038, and the Lyman lines, plus strong Lyman limit absorption at the same redshift. Fig. 1 shows our estimates of the continuum on either side of the Lyman edge (912 $\AA$ ). These continuum estimates are simple power laws of the form $F_{\lambda} \propto \lambda^{\alpha}$, with a Gaussian profile added at $\sim 1034 \AA$ in the quasar frame to account for the broad O VI $\lambda \lambda 1032$, 1038 emission line. The AALs and numerous unrelated Ly $\alpha$ forest lines make it difficult to locate the true continuum. We constrain our fits to using the median flux in narrow wavelength windows that appear relatively free of absorption lines. At wavelengths $>912 \AA$, the continuum slope near the Lyman edge is not well constrained due to absorption-line blending. We therefore consider three different continua (shown by the coloured curves in Fig. 1) that are 
upper and lower limits and a best-guess middle case based on visual inspection.

\section{ABSORPTION FEATURES}

\subsection{Line identifications}

Figs 2-6 show the AAL profiles for all four quasars on a velocity scale relative to the emission-line redshifts. All of the quasars have multiple or complex/blended AAL systems. We identify individual AAL systems starting with the C IV $\lambda 1548,1551$ and Si IV $\lambda 1394$, 1403 doublets because these lines are not contaminated in Ly $\alpha$ forest and they tend to be strong and well-measured at wavelengths with high signal-to-noise ratios in our spectra. If these lines are broad or blended with neighbouring features, we use weaker lines such as C II $\lambda 1335$ and Si II $\lambda 1260,1304$, and 1527, if available, to identify individual components and measure redshifts for the system. We then search other lines at these same redshifts. For Q0119-046, the search for additional lines includes the HST-FOS spectrum down to $\sim 750 \AA$ in the quasar rest frame. The lines identified in each quasar are listed in Tables $2-6$ below. We now describe the line measurements and the contents of these data tables.

\subsection{Line profile fits}

We fit each AAL with a Gaussian optical depth profile given by,

$\tau_{v}=\tau_{0} \mathrm{e}^{-v^{2} / b^{2}}$,

where $\tau_{v}$ is the optical depth at velocity $v$, and $b$ is the Doppler parameter. $\tau_{0}$ is the line centre optical depth, equal to

$\tau_{0}=\frac{\sqrt{\pi} e^{2}}{m_{e} c} \frac{N f \lambda_{0}}{b}$,

where $N$ is the column density, $f$ is the oscillator strength, and $\lambda_{0}$ is the laboratory line-centre wavelength. We assume for simplicity that the background light source has a uniform brightness and the absorbing medium is homogeneous, with the same optical depth along every sightline, so the observed intensity at velocity is

$\frac{I_{v}}{I_{0}}=1-C_{v}+C_{v} \mathrm{e}^{-\tau_{v}}$,

where $I_{0}$ is the continuum intensity, $I_{v}$ is the measured intensity at velocity $v, C_{v}$ is the covering fraction of the absorbing medium across the emission source, such that $0<C_{v} \leq 1$ (Barlow et al. 1997; Hamann et al. 1997a; Ganguly et al. 1999). It is known that actual absorbers in quasar spectra can be inhomogeneous, with a range of optical depths across the projected area of the emission source (Barlow et al. 1997; Hamann et al. 2001; Arav, Korista \& de Kool 2002; de Kool, Korista \& Arav 2002; Hamann \& Sabra 2004; Arav et al. 2005). In this situation, equation (3) yields spatially averaged optical depths and approximate covering fractions for material with $\tau_{v} \gtrsim 1$ (Hamann \& Sabra 2004). Real absorbers can also have velocity-dependent $C_{v}$ values that can differ between lines (Barlow \& Sargent 1997; Hamann et al. 1997a; Ganguly et al. 1999; Hamann et al. 2001; Hamann \& Sabra 2004). The velocitydependent effects are not important for the narrow absorption lines in our study. Therefore, for simplicity, we assume a constant covering fraction across the line profiles, e.g. $C_{v}=C_{0}$, but the value of $C_{0}$ can differ between lines. We also note that equation (3) applies generically to partial covering situations regardless of its origins, which might include scattered light or partial covering of the accretion-disc continuum source versus the broad emission line region (Ganguly et al. 1999; Hamann \& Sabra 2004). We discuss the origins of the partial covering further in Section 4.6.

To fit the lines in the low-resolution HST spectrum and the narrow AALs (velocity widths $\lesssim 30 \mathrm{~s}^{-1}$ ) in the Keck and VLT spectra, we convolve Gaussian optical depth profile with a Gaussian kernel that represents the instrumental broadening, specifically,

$\frac{I_{v}}{I_{0}}=\int \mathrm{e}^{-\tau_{v^{\prime}}} G\left(v-v^{\prime}\right) \mathrm{d} v^{\prime}$,

where $G(v)$ is Gaussian kernel defined by

$G(v)=\frac{1}{\sqrt{\pi} \Delta v_{\text {ins }}} \mathrm{e}^{-\frac{\Delta v^{2}}{\Delta v_{\text {ins }}^{2}}}$,

and $\Delta v_{\text {ins }}$ is the instrumental velocity resolution.

We derive estimates of $\tau_{0}$ and $C_{0}$ from equation (3) by fitting the lines in doublets and multiplets simultaneously with fixed optical depth ratios determined by atomic physics. The lines are also required to have the same redshift, Doppler parameter, column density, and covering fraction. However, the tradeoff between $\tau_{0}$ and $C_{0}$ in the measured line depths can lead to ambiguous results in limiting cases where $\tau_{0} \gg 1$ or $\tau_{0} \ll 1$, and where only a single line is available. Thus we consider four cases: (1) Heavily saturated lines based on the $\sim 1: 1$ observed depth ratio of the doublet and/or flat-bottom profiles that do not reach zero intensity, see for example components 2,7 and 11 of $\mathrm{C}$ IV $\lambda \lambda 1548,1551$ and component 2 of Ly $\alpha$ in Fig. 2. In these situations, equation (3) simplifies to $C_{0} \approx 1-I_{v} / I_{0}$, where the covering fraction equals the observed depth of the line, and we adopt a conservative minimum optical depth of $\tau_{0} \gtrsim 3$ in the weakest doublet/multiplet component. (2) Doublets or multiplets that appear unsaturated based on intermediate line ratios, e.g. between $2: 1$ and 1:1 for the doublets, ${ }^{2}$ such as component 2 of $\operatorname{SiIV} \lambda \lambda 1394,1403$ in Fig. 2). In this case, we use equations (1) and (3) to solve for both $C_{0}$ and $\tau_{0}$ by fitting the doublet lines simultaneously. Some examples of these fits and the partial covering determinations are described in Section 3.3.1 below (see also Fig. 7). (3) Rare weak doublets that appear to have $\tau_{0} \ll 1$ based on $\sim 2: 1$ strength ratios, such as components 2 of Si II $\lambda 1260,1527$ in Fig. 2 . In this case, the values of $\tau_{0}$ and $C_{0}$ are degenerate in equation (3) and cannot be determined separately. Thus we adopt $C_{0}=1$ to derive conservatively small lower limits on $\tau_{0}$ and the column densities. And (4) single lines that do not reach zero intensity, such as component 2 of $\mathrm{Si}$ III $\lambda 1206$ in Fig. 2. Here again we adopt $C_{0}=1$ to derive conservatively small lower limits on the column densities. We find partial covering situations in components 2, 3, 5, 7, and 11 in Q0119-046, and component 2 in Q0334-204. For Q0119-046, we perform a detailed discussion in Section 3.3.1 below. And for Q0334-204, only component 2 in C IV shows partial covering, which is not convincing. We do not perform a further partial covering analysis on this quasar.

Another complication is line blending. If the blending is moderate between two or more components in the same transition, such that distinct components with separate minima are apparent (e.g. components 2 and 3 of C IV $\lambda \lambda 1548,1551$ in Fig. 2), we fit all of the blended components simultaneously while allowing their $b$ values and centroids to be free parameters. If the blending is more severe,

\footnotetext{
${ }^{2}$ The optical depth ratios for lines sharing a common lower energy state are set by the ratio of their $f \lambda$ values, where $f$ is the oscillator strength and $\lambda$ is the line wavelength. This ratio is $\sim 2: 1$ for the doublets discussed in this paper, such as C IV $\tau(1548 \AA) / \tau(1551 \AA)$.
} 


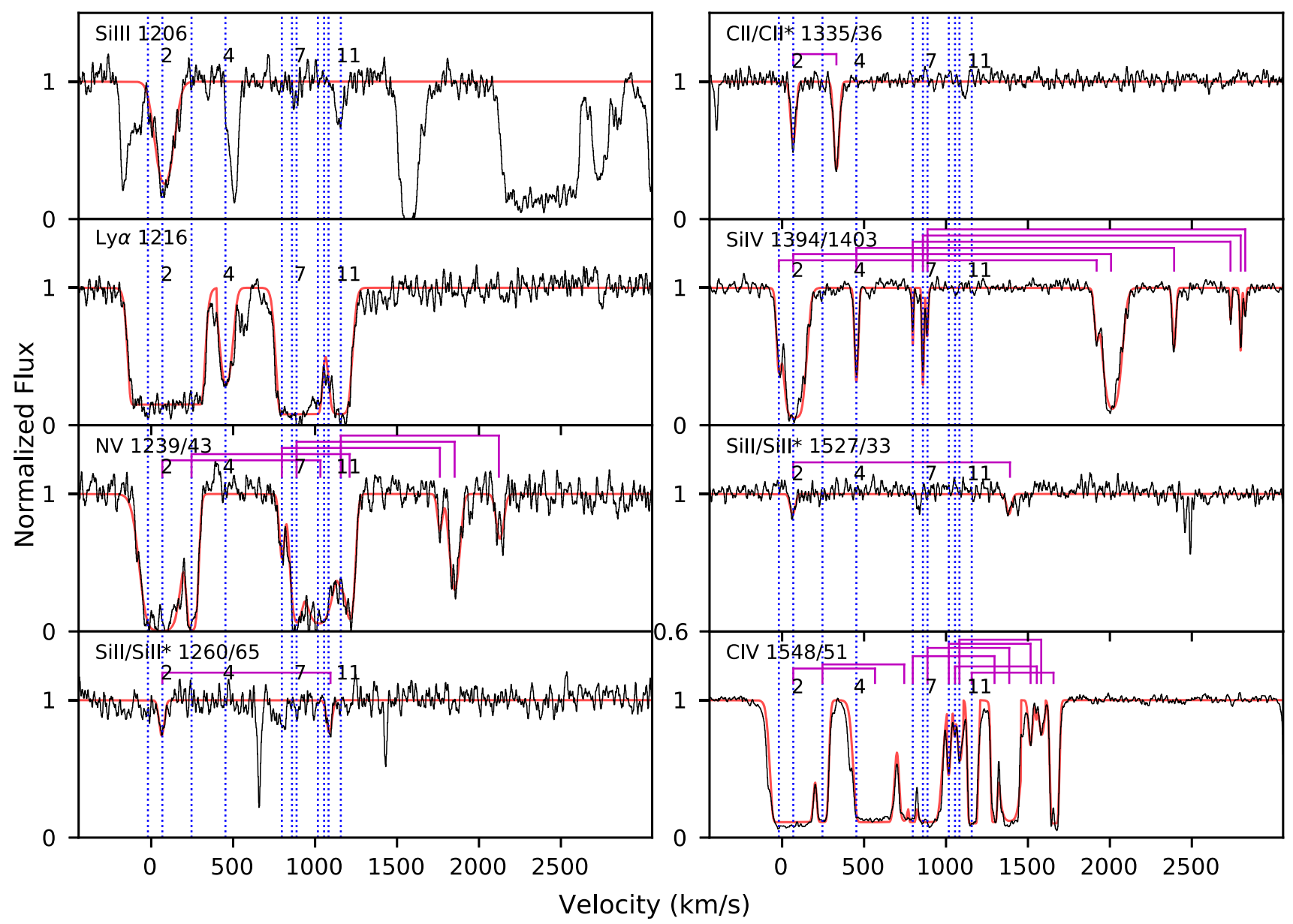

Figure 2. Q0119-046: Normalized line profiles in the Keck-HIRES spectrum plotted on a velocity scale relative to the quasar redshift (Table 1). The spectra are shown in black, and the fitting lines are shown in red. The blue dash lines are components from 1 to 11, and the brackets show the doublets. The velocities pertain to the short-wavelength lines in the doublets.

such that the observed feature forms a single line (e.g. components 1, 2, and 3 of Ly $\alpha$ in Fig. 2), we adopt $b$ values and centroid wavelengths determined from fits to other unblended lines of similar ionized ions in the same redshift system (if available), then fit the multiple lines in the blend simultaneously to derive their separate column densities and associated errors. Finally, if the blending involves unrelated lines (from different ion or features in the Lyman forest, e.g. component $7^{*}$ of $\mathrm{N}$ III $\lambda 990$ in Fig. 3 and component 4 of Nv $\lambda 1243$ in Fig. 6), we again adopt $b$ values and centroid wavelengths determined from fits to other unblended lines of similar ionized ions in the same redshift system, and then fit the line to obtain a column density or maximum column density, depending of the severity of the blending.

Tables 2-6 list the derived line fit parameters and associated uncertainties. Footnotes in these tables indicate the procedure used for fitting the lines and deriving or assuming values of $C_{0}, \tau_{0}$, and $N$, while the notes provide additional information on blends. The line data are organized in the tables according to the redshifts, i.e. component numbers in the first column as labelled in Figs 2-6. The uncertainties listed for most of the parameters are $1 \sigma$ errors derived from the fits, affected mainly by pixel-to-pixel noise fluctuations in the spectra. They do not consider errors in the continuum placement. For very optically thick lines based on $\sim 1: 1$ doublet ratios, we can obtain only lower limits on the column densities. We derive these limits from equation (2) using $b$ values from our fits with $\tau_{0} \gtrsim 3$. If the saturated lines are blended with neighbouring systems to make their $b$ values uncertain (e.g. components 1,2 , and 3 of Ly $\alpha$ in Fig. 2), we derive column density limits by combining $\tau_{0} \gtrsim 3$ with $b$ values measured from other unblended lines of similar ions in the same redshift system, as marked in the notes in the tables.

Finally, for the quasars Q0105+061, Q0334-204, and Q2044-168, we estimate upper limits on excited-state lines not detected, such as $\mathrm{Si}_{\text {II* }}^{*} 1533$ and $\mathrm{C}_{\text {II }}^{*} \lambda 1336$, if the corresponding resonance lines, e.g. Si II $\lambda 1527$ and C II $\lambda 1335$, are cleanly measured. We use $b$ values and redshifts from the resonance lines to manually draw synthetic line profiles at the excited-state line positions and thereby determine $\sim 3 \sigma$ upper limits on the excited-state column densities.

\subsection{Q0119-046}

Q0119-046 is a special case because it has a rich complex of blended AALs and additional wavelength coverage from HST that includes Lyman limit absorption and AALs measured at lower spectral resolution.

\subsubsection{Absorption lines}

We identify 11 distinct AAL redshift systems in the Keck spectrum (Fig. 2), but only 4 systems in the HST spectrum due to the lower 


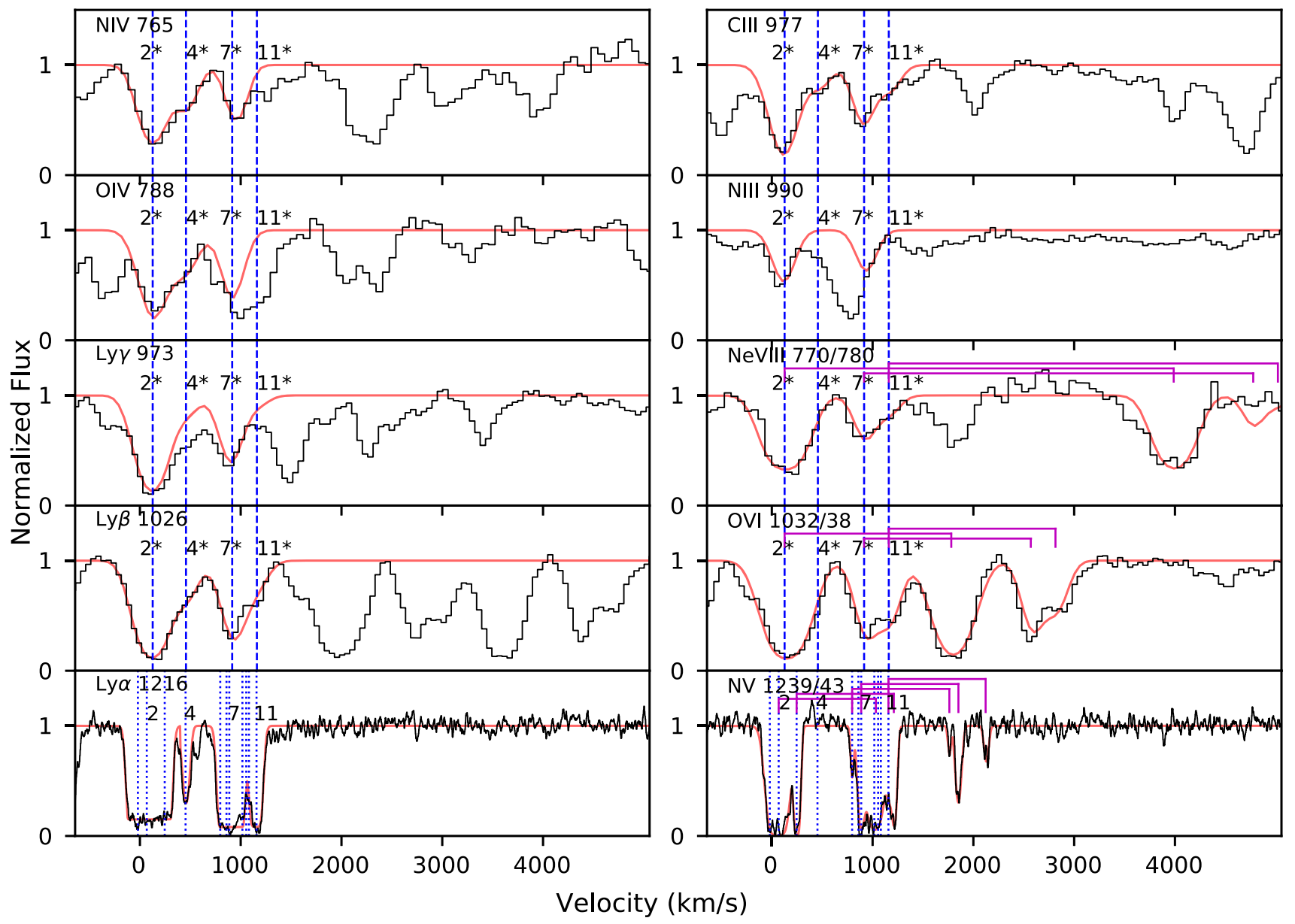

Figure 3. Q0119-046: Normalized line profiles in the HST-FOS spectrum plotted on a velocity scale relative to the quasar redshift (Table 1). The spectra are shown in black, and the fitting lines are shown in red. The blue dash lines are components, and the brackets show the doublets. The velocities pertain to the short-wavelength lines in the doublets. The bottom two panels are Ly $\alpha$ and $\mathrm{N} v$ spectra from Keck in comparison with the components of HST spectra.

resolution and blending (Fig. 3). We label the components in the $H S T$ spectrum $2^{*}, 4^{*}, 7^{*}$, and $11^{*}$ to identify them loosely with the strongest components 2, 4, 7, and 11 in the Keck spectrum. Fig. 3 shows important AALs such as O VI, Ne VIII, and some Lyman lines in the HST spectrum compared to C IV and N v measured in the Keck spectrum. The Keck spectra in Fig. 2 show that higher ionization lines (such as C IV) and stronger transitions (e.g. Ly $\alpha$ ) are broader and more blended. These lines also appear to be saturated based on $\sim 1$ : 1 doublet ratios and/or flat-bottomed profiles that do not reach zero intensity. The depths of these saturated lines yield the covering fractions (Section 3.2).

Other lines require closer examination. Fig. 7 shows a partial covering analysis for components 2 and $2 *$ in the doublets Si IV $\lambda \lambda 1394$, 1403, O VI $\lambda \lambda 1032,1038$, and Ne VIII $\lambda \lambda 770,780$. Specifically, fits to the stronger short-wavelength lines in these doublets are used to predict the strength of the weaker long-wavelength lines assuming $C_{0}=1$. The predicted strengths are too weak in all cases, indicating $C_{0}<1$ with specific $C_{0}$ values listed in Tables 2 and 3 . There might be some ambiguity about these results for O VI and Ne VIII because these lines are not well resolved in the HST spectrum. However, the comparisons to the well-resolved N v lines in the Keck spectrum shown in Fig. 3, and the tendency throughout the Q0119-046 spectrum for higher ion AALs to be broader and smoother (Fig. 2) indicates that the $\mathrm{O}$ VI and $\mathrm{Ne}$ VIII lines are reasonably resolved and not composed of narrow saturated features that could mimic partial covering at the HST spectral resolution. There is another possibility that the long-wavelength doublet components in O VI and Ne VIII are made stronger to mimic partial covering due to blending with unrelated absorption line the Ly $\alpha$ forest. However, there is no evidence for significant blending because the long-wavelength lines in both ions have redshifts and profiles as predicted from the shortwavelength lines. We conclude that significant partial covering does occur for $\mathrm{O}$ VI and $\mathrm{Ne}$ VIII, with $C_{0}$ values given in Tables 2 and 3.

\subsubsection{Lyman limit and Lyman series}

The Lyman limit in Q0119-046 provides an accurate measurement of the H I column density and another estimate of the H I covering fraction. We fit the Lyman limit absorption using equation (3) with optical depths given by

$\tau_{\lambda}=6.63 \times 10^{-18} N(\mathrm{HI})\left(\frac{\lambda}{912 \AA}\right)^{3}$,

where $N(\mathrm{HI})$ is the $\mathrm{H}$ I column density in $\mathrm{cm}^{-2}$ (Osterbrock 1989). The main uncertainty in this analysis is the unabsorbed continuum intensity, $I_{0}$. For this we extrapolate our best-fitting power law at $\lambda>912 \AA$ with $\alpha=-1.6$ (Section 2.2.1 and Fig. 1) to shorter wavelengths, and we use the range of possible values from $\alpha=$ -1.2 to -2.0 to assess the uncertainties. We also fix the redshift 

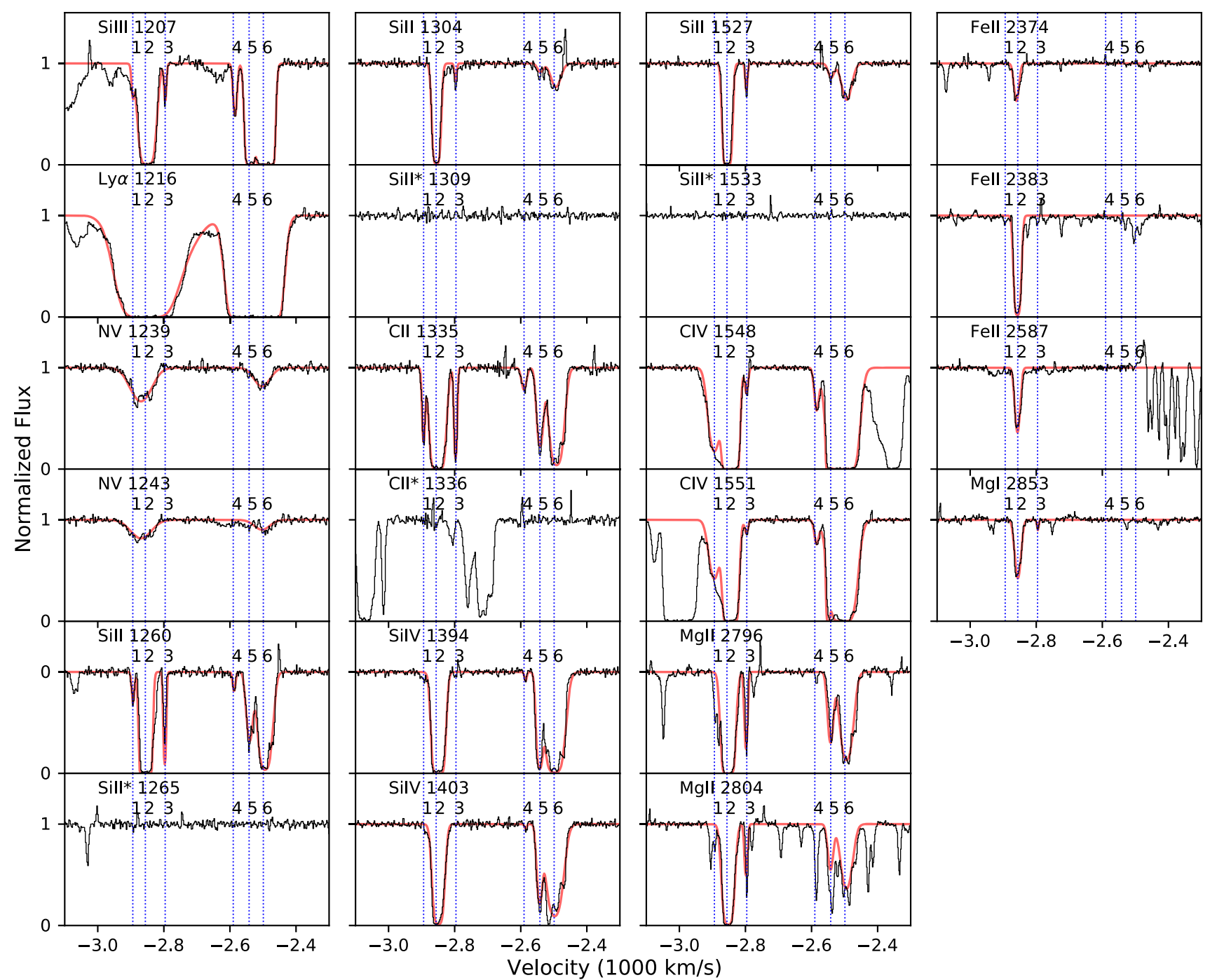

Figure 4. Q0105+061: Normalized line profiles in the VLT-UVES spectrum plotted on a velocity scale relative to the quasar redshift (Table 1). The spectra are shown in black, and the fitting lines are shown in red. The blue dash lines are components.

to that of component $2^{*}$ (Table 3 ) because this system includes many strong Lyman lines and it is the only system with an $\mathrm{HI}$ column density large enough to produce a Lyman limit (based on our absorption-line assessments). We divide by this power law and then fit the resulting normalized continuum at $\lambda<912 \AA$ using equations (3) and (6), constrained by the median flux in narrow wavelength intervals that appear unaffected by Ly $\alpha$ forest lines.

The results (shown in Fig. 8) indicate a covering fraction $C_{0}=$ $0.92 \pm 0.02$ and column density $\log N(\mathrm{HI})\left(\mathrm{cm}^{-2}\right)=17.68 \pm 0.04$ based our best-fitting continuum with $\alpha=-1.6$. Additional fits using the full range of continua from Fig. $1(\alpha=-1.2$ to -2.0$)$ yield firm lower and upper limits $C_{0}=0.90\left[\log N(\mathrm{HI})\left(\mathrm{cm}^{-2}\right)=\right.$ 17.78] and $C_{0}=0.95\left[\log N(\mathrm{HI})\left(\mathrm{cm}^{-2}\right)=17.60\right]$, respectively. These covering fractions are consistent with the upper lines in the Lyman series (component $2^{*}$ in Fig. 3) but slightly larger than the well-determined value of $C_{0}=0.85$ for Ly $\alpha$ (Fig. 2, Table 2). We attribute the anomaly in Ly $\alpha$ to its location on top of strong and broad Ly $\alpha$ emission line, indicating that the Ly $\alpha$ absorber only partially covers the broad emission-line region (see Section 4.6 for more discussion). We use a Gaussian function to model the Ly $\alpha$ emission line and try to eliminate its effect. But our model is only a lower limit due to the severe contamination by the strong absorptions lines. This lead to a smaller value of $C_{0}$ for Ly $\alpha$.

These results for $C_{0}$ and $\log N(\mathrm{HI})$ from the Lyman limit are more reliable than what can be determined from Lyman series lines because the lines are underresolved in the HST spectrum and they are contaminated by the Ly $\alpha$ forest. Therefore, we adopt $C_{0}=$ 0.92 and $\log N(\mathrm{HI})\left(\mathrm{cm}^{-2}\right)=17.68$ and apply our fitting procedure to the upper Lyman series lines to determine that they have $b=$ $81 \pm 10 \mathrm{~s}^{-1}$ (see component $2^{*}$ in Fig. 3).

\section{ANALYSIS}

In this section, we analyse the AAL systems that have resonance lines of $\mathrm{C}$ II or $\mathrm{Si}$ II detected so that our measurements or upper limits on the corresponding excited-state lines, $\mathrm{C} \mathrm{II}^{*}$ or Si II*, provide density and location constraints on the absorption environments. This includes component 2 in Q0119-046, all components in Q0105+061, components 1, 2, and 3 in Q0334-204, and all components in Q2044-168. These AAL systems also include multiple ions of the same element, such as C II/C IV and $\mathrm{Si} \mathrm{II/Si} \mathrm{III/Si} \mathrm{IV,}$ that we use to estimate the ionizations, total column densities, and 


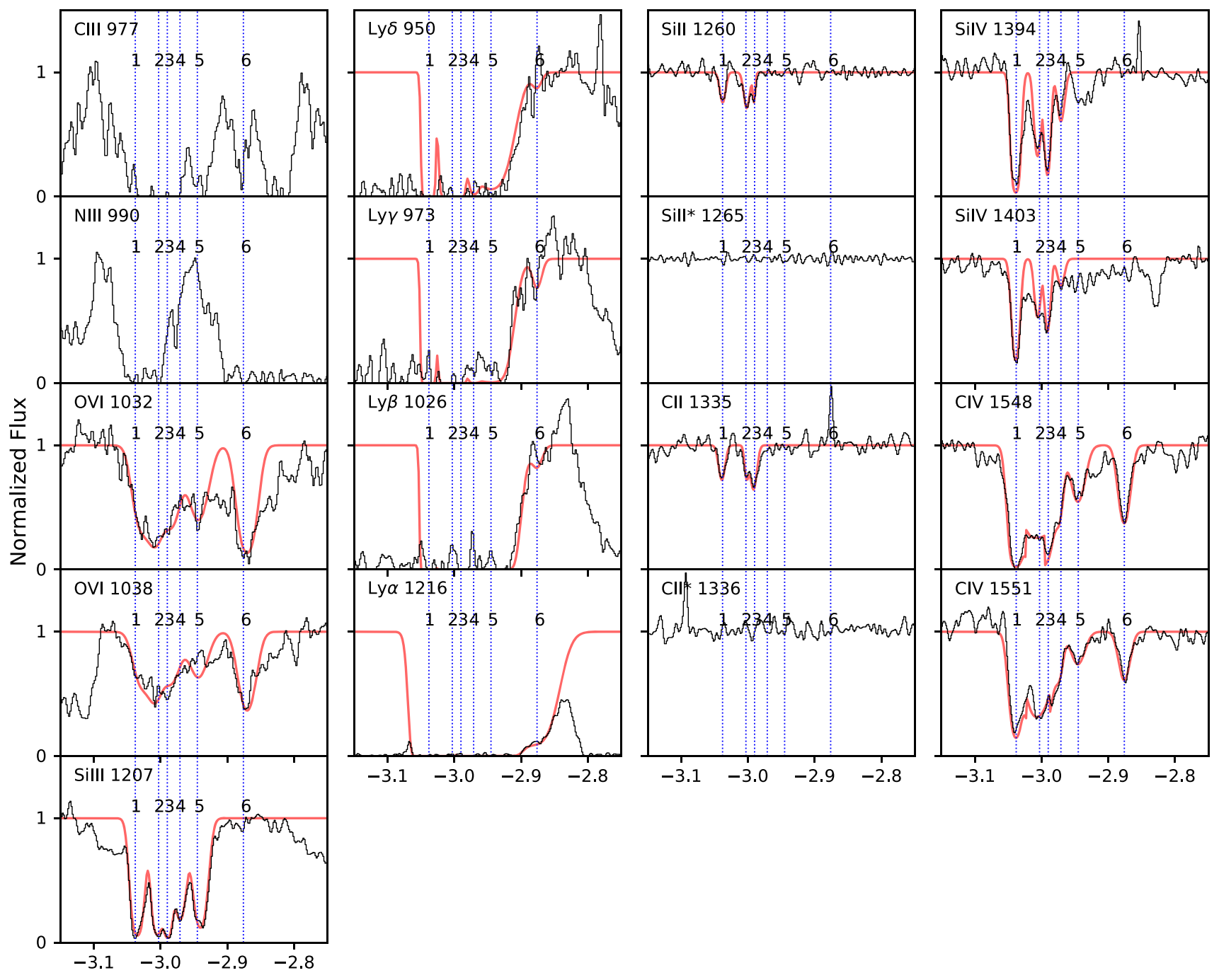

Velocity $(1000 \mathrm{~km} / \mathrm{s})$

Figure 5. Q0334-204: Normalized line profiles in the VLT-UVES spectrum plotted on a velocity scale relative to the quasar redshift (Table 1). The spectra are shown in black, and the fitting lines are shown in red. The blue dash lines are components.

element abundances compared to photoionization models. Table 7 lists various derived parameters for these systems, which we discuss below.

\subsection{Electron number density}

C II and Si II have similar energy level structures with a ground ${ }^{2} \mathrm{P}^{o}$ term that is split into a true ground $J=1 / 2$ state and a slightly excited $J=3 / 2$ state. This leads to pairs of $\mathrm{C} \mathrm{II} * / \mathrm{C}$ II and $\mathrm{Si}$ II*/Si II lines whose ratios are density dependent because the excited $J=3 / 2$ states are populated mainly by collisions from the $J=1 / 2$ ground (Bahcall \& Wolf 1968; Sargent et al. 1982; Morris et al. 1986; Hamann et al. 2001; Dunn et al. 2010; Arav et al. 2013; Finn et al. 2014). For a simple two-level atom where the excited-state energy is small compared to $k T$, the density dependence can be written as

$n_{\mathrm{e}}=n_{\text {cr }}\left(\frac{N_{\text {lo }}}{N_{\text {up }}} \frac{g_{\text {up }}}{g_{\text {lo }}}-1\right)^{-1}$

where $n_{\mathrm{e}}$ is the electron density, $n_{\mathrm{cr}}$ is the critical electron density of the upper state, $N_{\text {lo }}$ and $N_{\text {up }}$ are the column densities in the ground and excited states, respectively, and $g_{\text {up }} / g_{10}=2$ is the ratio of their statistical weights. For a temperature of $10^{4} \mathrm{~K}$, we derive $n_{\mathrm{cr}} \approx$ $1766 \mathrm{~cm}^{-3}$ for the Si II upper state and $n_{\mathrm{cr}} \approx 49 \mathrm{~cm}^{-3}$ for C II [using collision strengths from Tayal (2008a,b) and radiative decay rates from the NIST atomic spectra database ${ }^{3}$ (Kramida et al. 2018)].

Table 7 shows the electron densities in $\mathrm{Si}$ II or $\mathrm{C}$ II regions that result from equation (7) combined with the column density data in Tables 2-6. The excited-state lines are detected only in component 2 of Q0119-046. The density listed for Q0119-046 derives from the $N\left(\mathrm{Si} \mathrm{II}{ }^{*}\right) / N\left(\mathrm{Si}\right.$ II) with the result $\log n_{\mathrm{e}}\left(\mathrm{cm}^{-3}\right)=3.4 \pm 0.3$. This is consistent with the observed ratio of $N\left(\mathrm{C}\right.$ II $\left.^{*}\right) / N(\mathrm{C}$ II $)$ near 2 , which yields only a lower $\operatorname{limit} \log n_{\mathrm{e}}\left(\mathrm{cm}^{-3}\right) \gtrsim 2.2$ (consistent with Sargent et al. 1982).

All of the other quasars with non-detections provide only upper limits on the densities. The upper limits listed for components 2, 3, 5 , and 6 in Q0105+061 are based on $N\left(\mathrm{Si} \mathrm{II}^{*}\right) / N(\mathrm{Si}$ II) because we fit multiple Si II lines (1260, 1304, and $1527 \AA$ A) simultaneously with the

\footnotetext{
${ }^{3}$ http://www.nist.gov/pml/data/asd.cfm
} 

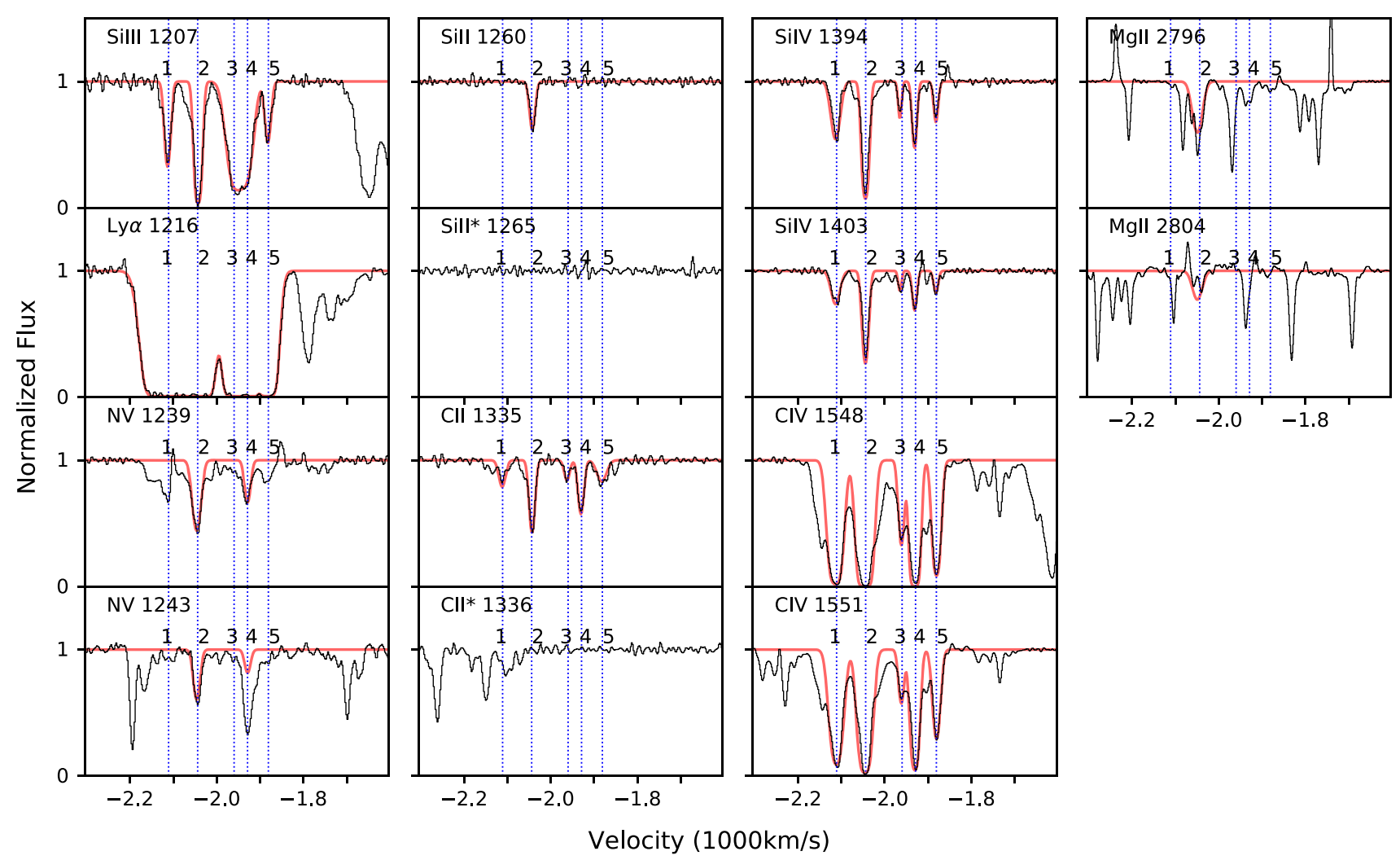

Figure 6. Q2044-168: Normalized line profiles in the VLT-UVES spectrum plotted on a velocity scale relative to the quasar redshift (Table 1). The spectra are shown in black, and the fitting lines are shown in red. The blue dash lines are components.

same $b$ parameter, velocity shift, and column density, which lead to reasonably good constraints. The upper limits listed for components 1 and 4 in Q0105+061 are based on $N\left(\mathrm{C} \mathrm{II}^{*}\right) / N(\mathrm{C} \mathrm{II})$ because Si II lines are weak and only $\mathrm{Si}$ II $\lambda 1260$ is detected. The densities listed for Q0334-204 and Q2044-168 derive from $N\left(\mathrm{C} \mathrm{II}^{*}\right) / N(\mathrm{C}$ II $)$ because the resonance $C_{\text {II }} \lambda 1335$ lines are well measured and this ratio provides smaller upper limits than $N\left(\mathrm{Si} \mathrm{II}^{*}\right) / N(\mathrm{Si}$ II $)$. The upper limits in these quasars range from $\lesssim 150$ to $\lesssim 15 \mathrm{~cm}^{-3}$.

\subsection{Photoionization models}

We assume that the absorbers are in photoionization equilibrium with the quasar radiation field and examine their ionization properties using the computer code CLOUDY (version 13.03, Ferland et al. 2013). The calculations use a plane-parallel absorbing geometry, solar abundances, and a constant gas density $n_{\mathrm{H}}=100 \mathrm{~cm}^{-3}$. The specific value of the density is not important for our analysis of the ground-state column densities (Hamann 1997; Hamann et al. 2002; Leighly, Dietrich \& Barber 2011). Recent work by Baskin, Laor \& Stern (2014) favours constant pressure absorbing clouds instead of constant density for BAL outflows. However, experiments with CLOUDY show that this also does not have an impact on our results. The ionization structure does depend on the shape and intensity of the incident spectral energy distribution (SED). We use an SED that is roughly characteristic of luminous quasars (Reeves \& Turner 2000; Richards et al. 2006; Hopkins, Richards \& Hernquist 2007; Shull, Stevans \& Danforth 2012). It is described by a power law, $f_{v} \propto v^{\alpha}$, with spectral indices $\alpha_{\mathrm{UV}}=-0.5$ and $\alpha_{\mathrm{X}}=-0.9$ at UV and X-ray wavelengths, respectively. These segments are normalized to yield the two-point power-law index $\alpha_{\mathrm{OX}}=-1.7$ between $2500 \AA$ and $2 \mathrm{keV}$ (Hamann et al. 2013). The UV and $\mathrm{X}$-ray segments connect smoothly using a Planck exponential with temperature $350000 \mathrm{~K}$. We specify the intensity of this radiation field using the ionization parameter, $U$, which is defined as the dimensionless ratio between the number density of hydrogen-ionizing photons at the illuminated face of the clouds to the number density of hydrogen atoms, e.g.

$U \equiv \frac{\Phi(\mathrm{H})}{n_{\mathrm{H}} c}$,

where $n_{\mathrm{H}}$ is the total hydrogen density, $c$ is the speed of light, and $\Phi(\mathrm{H})$ is the flux of H-ionizing photons given by

$\Phi(\mathrm{H})=\frac{1}{4 \pi R^{2}} \int_{v_{1}}^{\infty} \frac{L_{v}}{h v} \mathrm{~d} v$,

where $L_{v}$ is the quasar luminosity density, $R$ is the radial distance between the absorber and the quasar, and $h \nu_{1}=13.6 \mathrm{eV}$.

We run the CLOUDY simulations with a fixed HI column density $\log N(\mathrm{HI})\left(\mathrm{cm}^{-2}\right)=17.7$ and a total column density $\log N(\mathrm{H})\left(\mathrm{cm}^{-2}\right)=20.9$ for Q0119-046 based on our measurements in Section 3.3.2 (Tables 2 and 3) and Section 4.3 below. For the other three quasars, we adopt $\log N(\mathrm{HI})\left(\mathrm{cm}^{-2}\right)=16$ consistent with the lower limits listed in Tables $4-6$. We adopt $\log N(\mathrm{HI})\left(\mathrm{cm}^{-2}\right)=16$ to illustrate the results in a regime where the clouds are optically thin in the Lyman continuum such that the calculated ionizations do not depend on the specific value of $N(\mathrm{HI})$. The actual H I column densities are not known for these quasars. We discuss other possibilities in Section 4.3 below. 
Table 2. Individual absorption lines of Q0119-046 (Keck spectrum). Columns show component number, absorption redshift ( $\left.z_{\text {abs }}\right)$ and the corresponding velocity shift $(v)$ relative to the emission-line redshift, line identification, and rest wavelength, observation wavelength, Doppler $b$ parameter, logarithm of column density, covering fraction, and notes [sat $=$ saturated line, $\mathrm{bl}=$ blended with neighbouring systems, unbl $=$ blended with unrelated lines $(\mathrm{e} . \mathrm{g}$. lines in the Lyman forest), $\mathrm{w}=$ weak line]. For Ly $\alpha$ lines, we obtain $b$ and $N(\mathrm{HI})$ values from the fits of the Lyman limit and Lyman series, which is described in Section 3.3.2.

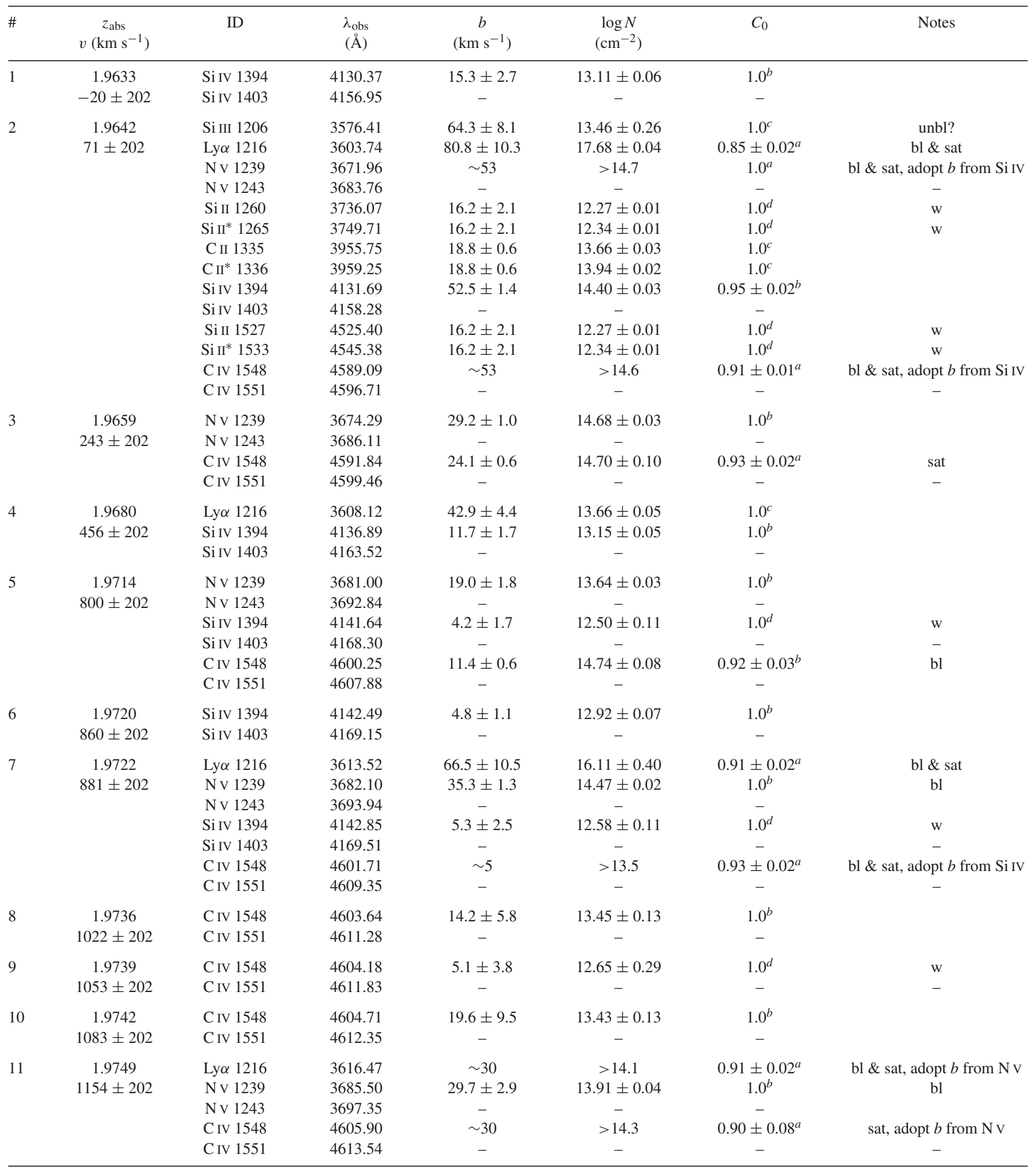

\footnotetext{
${ }^{a}$ Heavily saturated lines, whose $C_{0}$ equals the observed depth of the line.

${ }^{b}$ Unsaturated doublets, where we solve for $C_{0}$ using equation (3).

${ }^{c}$ Unsaturated single lines, where we adopt $C_{0}=1$ because it is not constrained.

${ }^{d}$ Weak doublets, where we take a conservative approach by setting $C_{0}=1$.
} 
Table 3. Individual absorption lines of Q0119-046 (HST spectrum). See Table 2 for descriptions of the table contents. For the notes, nl = no obvious lines, $\mathrm{bl}=$ blended with neighbouring systems, unbl = blended with unrelated lines (e.g. lines in the Lyman forest).

\begin{tabular}{|c|c|c|c|c|c|c|c|}
\hline$\#$ & $\begin{array}{c}z_{\mathrm{abs}} \\
v\left(\mathrm{~km} \mathrm{~s}^{-1}\right)\end{array}$ & ID & $\begin{array}{l}\lambda_{\text {obs }} \\
(\AA)\end{array}$ & $\begin{array}{c}b \\
\left(\mathrm{~km} \mathrm{~s}^{-1}\right)\end{array}$ & $\begin{array}{c}\log N \\
\left(\mathrm{~cm}^{-2}\right)\end{array}$ & $C_{0}$ & Notes \\
\hline $2^{*}$ & $\begin{array}{c}1.9646 \\
111 \pm 202\end{array}$ & $\begin{array}{l}\text { N IV } 765 \\
\text { Ne VIII } 770 \\
\text { Ne VIII } 780 \\
\text { O IV } 788 \\
\text { Ly } \gamma 973 \\
\text { C III } 977 \\
\text { N III } 990 \\
\text { Ly } \beta 1026 \\
\text { O VI } 1032 \\
\text { O VI } 1037\end{array}$ & $\begin{array}{l}2268.60 \\
2284.05 \\
2313.47 \\
2335.84 \\
2883.40 \\
2896.04 \\
2933.75 \\
3040.69 \\
3059.38 \\
3076.33\end{array}$ & $\begin{array}{c}117 \pm 28 \\
147 \pm 18 \\
- \\
110 \pm 19 \\
80.8 \pm 10.3 \\
111 \pm 22 \\
72.5 \pm 24.1 \\
80.8 \pm 10.3 \\
172 \pm 41 \\
-\end{array}$ & $\begin{array}{c}14.74 \pm 0.16 \\
16.43 \pm 0.38 \\
- \\
15.53 \pm 0.14 \\
17.68 \pm 0.04 \\
14.61 \pm 0.17 \\
14.08 \pm 0.03 \\
17.68 \pm 0.04 \\
15.92 \pm 0.31 \\
-\end{array}$ & $\begin{array}{c}1.0^{a} \\
0.68 \pm 0.07^{b} \\
- \\
1.0^{a} \\
0.92 \pm 0.02^{c} \\
1.0^{a} \\
1.0^{a} \\
0.92 \pm 0.02^{c} \\
0.90 \pm 0.08^{b} \\
-\end{array}$ & \\
\hline $4^{*}$ & 1.9680 & $\begin{array}{l}\text { N IV } 765 \\
\text { O IV } 788 \\
\text { Ly } \gamma 973 \\
\text { C III } 977 \\
\text { Ly } \beta 1026\end{array}$ & $\begin{array}{l}2270.53 \\
2337.89 \\
2886.49 \\
2899.79 \\
3044.34\end{array}$ & $\begin{aligned} 98.9 & \pm 44.1 \\
101.9 & \pm 51.0 \\
& \sim 43 \\
& \sim 100 \\
& \sim 43\end{aligned}$ & $\begin{array}{c}14.04 \pm 0.10 \\
14.86 \pm 0.17 \\
<14.8 \\
13.33 \pm 0.28 \\
<14.8\end{array}$ & $\begin{array}{l}1.0^{a} \\
1.0^{a} \\
1.0^{a} \\
1.0^{a} \\
1.0^{a}\end{array}$ & $\begin{array}{c}\text { bl } \\
\text { bl } \\
\text { unbl, adopt } b \text { from Ly } \alpha \\
\text { bl, adopt } b \text { from N IV } \\
\text { unbl, adopt } b \text { from Ly } \alpha\end{array}$ \\
\hline $11^{*}$ & $\begin{array}{c}1.9749 \\
1154 \pm 202\end{array}$ & $\begin{array}{l}\text { Ne VIII } 770 \\
\text { Ne VIII } 780 \\
\text { Ly } \gamma 973 \\
\text { C III } 977 \\
\text { Ly } \beta 1026 \\
\text { O VI } 1032 \\
\text { O VI } 1037\end{array}$ & $\begin{array}{c}2291.95 \\
- \\
2893.20 \\
2906.53 \\
3051.42 \\
3069.86 \\
3086.88\end{array}$ & $\begin{aligned} & \sim 45 \\
& - \\
& \sim 30 \\
& \sim 30 \\
& \sim 30 \\
44.6 & \pm 12.4 \\
& -\end{aligned}$ & $\begin{array}{c}<14.4 \\
- \\
14.71 \pm 0.32 \\
13.68 \pm 0.31 \\
14.71 \pm 0.32 \\
15.18 \pm 0.52 \\
-\end{array}$ & $\begin{array}{c}1.0^{a} \\
- \\
1.0^{a} \\
1.0^{a} \\
1.0^{a} \\
1.0^{b} \\
-\end{array}$ & $\begin{array}{l}\text { bl, adopt } b \text { from O VI } \\
\text { nl } \\
\text { bl, adopt } b \text { from Ly } \alpha \\
\text { bl, adopt } b \text { from Ly } \alpha \\
\text { bl, adopt } b \text { from Ly } \alpha\end{array}$ \\
\hline
\end{tabular}

${ }^{a}$ Unsaturated single lines, where we adopt $C_{0}=1$ because it is not constrained.

${ }^{b}$ Unsaturated doublets, where we solve for $C_{0}$ using equation (3).

${ }^{c}$ We adopt $C_{0}$ and $N$ from fits of the Lyman limit, described in Section 3.3.2.

\subsection{Ionization and total column densities}

Figs 9-12 show predicted ionization fractions for the well-measured AAL systems we discuss below. These predictions compared to the measured column density ratios in various ion pairs $(\mathrm{C} I / / \mathrm{C} \mathrm{IV}$, $\mathrm{Si}$ II/Si IV, Si II/Si III, etc.) yield estimates of the ionization parameter $U$ (shown by vertical lines in the figures) and its uncertainties (horizontal bars, based on the column density uncertainties in Tables 2-6). If the column density ratios of similar ion pairs (e.g. $\mathrm{C}$ II/C IV and $\mathrm{Si}$ II/Si IV) are consistent with a single $U$ value, we derive a weighted mean $U$ and weighted error. The results are listed in Table 7.

The temperature is the most important parameter in the far-UV. We ran additional CLOUDY models with the temperatures 175000 and $700000 \mathrm{~K}$, which might be considered extreme $( \pm 3 \sigma)$ deviations from the continuum we adopted (Section 4.2). Generally, we found the ionization parameter $\log U$ in the models changed by $\lesssim 0.1$ if considering $1 \sigma$ deviation, which is less than or similar to other uncertainties in the measured values of $\log U$.

Many of the systems in our sample exhibit a range of ions, e.g. from $\mathrm{Mg}$ I, Si II, C II, and Mg II up to N V, O VI, and Ne VIII, that cannot coexist spatially in the same absorber. In principle, these observed lines could form in the same clouds at a single $U$ if the column densities are large enough to radiatively shield the lower ions behind a thick layer of ionized gas. However, in component 2 of Q0119-046, the amount of shielding is well constrained by our measurement of $N(\mathrm{HI})$, e.g. the Lyman limit shown in Figs 1 and 8. Our CLOUDY simulations show that this produces minimal shielding with minimal effects on the ionization structure. Therefore, a range in $U$ is required.

The two $U$ values listed for Q0119-046 in Table 7 illustrate the range. They derive from the column density ratio $\mathrm{Si}$ II/Si III, which yields $\log U \sim-1.9$, up to $\mathrm{O}$ VI/Ne VIII, which yields $\log U \sim 0.9$ (assuming solar O/Ne abundances, see Fig. 9). If the Ne VIII absorber is at the same distance from the quasar as the low-ionization Si IIabsorbing region, then the range in $U$ values implies that the Ne VIII gas is $\sim 630$ times less dense than the Si II region (i.e. $n_{\mathrm{e}} \sim 4 \mathrm{~cm}^{-3}$ compared to $\sim 2500 \mathrm{~cm}^{-3}$ for $\mathrm{Si}$ II).

It is necessary to note that there is a large difference in the Doppler $b$ parameters between Si III $\left(b \sim 64 \mathrm{~s}^{-1}\right)$ and Si II $\left(b \sim 16 \mathrm{~s}^{-1}\right)$. The large difference indicates that the lines sample different physical regions in the absorber or, perhaps, that the Si III line is blended with a feature in the Ly $\alpha$ forest. We note that the Si III line width is similar 
Table 4. Individual absorption lines of Q0105+061. See Table 2 for descriptions of the table contents. For the notes, nl $=$ no obvious lines, sat $=$ saturated line, $\mathrm{bl}=$ blended with neighbouring systems, $\mathrm{w}=$ weak line, $\mathrm{unbl}=$ blended with unrelated lines (e.g. lines in the Lyman forest).

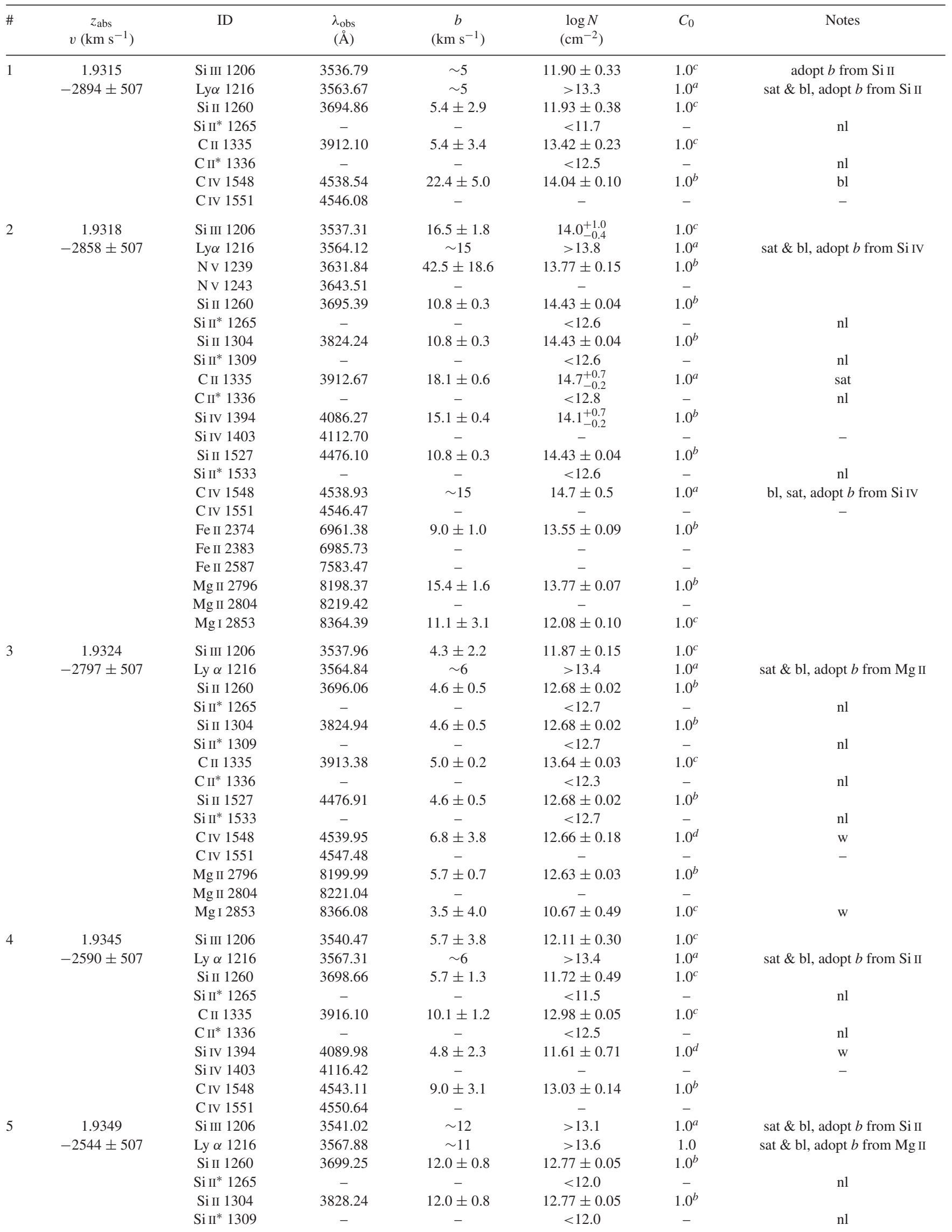


Table 4 - continued

\begin{tabular}{|c|c|c|c|c|c|c|c|}
\hline \# & $\begin{array}{c}z_{\mathrm{abs}} \\
v\left(\mathrm{~km} \mathrm{~s}^{-1}\right)\end{array}$ & ID & $\begin{array}{l}\lambda_{\text {obs }} \\
(\AA)\end{array}$ & $\begin{array}{c}b \\
\left(\mathrm{~km} \mathrm{~s}^{-1}\right)\end{array}$ & $\begin{array}{c}\log N \\
\left(\mathrm{~cm}^{-2}\right)\end{array}$ & $C_{0}$ & Notes \\
\hline & & C II 1335 & 3916.75 & $11.4 \pm 0.4$ & $13.82 \pm 0.02$ & $1.0^{c}$ & \\
\hline & & C II* 1336 & - & - & $<12.3$ & - & $\mathrm{nl}$ \\
\hline & & Si IV 1394 & 4090.46 & $7.1 \pm 0.5$ & $13.45 \pm 0.05$ & $1.0^{b}$ & \\
\hline & & Si IV 1403 & 4116.92 & - & - & - & \\
\hline & & Si II 1527 & 4480.78 & $12.0 \pm 0.8$ & $12.77 \pm 0.05$ & $1.0^{b}$ & \\
\hline & & Si II* 1533 & - & - & $<12.0$ & - & $\mathrm{nl}$ \\
\hline & & Mg II 2796 & 8207.05 & $10.6 \pm 0.9$ & $12.85 \pm 0.1$ & $1.0^{c}$ & \\
\hline & & Mg II 2804 & 8228.12 & - & - & - & unbl \\
\hline \multirow[t]{14}{*}{6} & 1.9353 & Si III 1206 & 3541.57 & $\sim 20$ & $>13.3$ & $1.0^{a}$ & sat $\&$ bl, adopt $b$ from $\mathrm{Si}$ II \\
\hline & $-2503 \pm 507$ & Ly $\alpha 1216$ & 3568.41 & $\sim 21$ & $>14.0$ & $1.0^{a}$ & sat $\& \mathrm{bl}$, adopt $b$ from $\mathrm{Mg}$ II \\
\hline & & N v 1239 & 3636.32 & $32.5 \pm 12.5$ & $13.36 \pm 0.06$ & $1.0^{b}$ & \\
\hline & & Si II* 1309 & - & - & $<12.5$ & - & $\mathrm{nl}$ \\
\hline & & C II 1335 & 3917.41 & $20.6 \pm 0.5$ & $14.41 \pm 0.02$ & $1.0^{c}$ & \\
\hline & & C II 1336 & - & - & $<12.3$ & - & $\mathrm{nl}$ \\
\hline & & Si IV 1394 & 4091.07 & $25.2 \pm 5.4$ & $14.0_{-0.2}^{+0.7}$ & $1.0^{b}$ & \\
\hline & & Si IV 1403 & 4117.53 & - & - & - & \\
\hline & & Si II 1527 & 4481.49 & $19.7 \pm 0.6$ & $13.46 \pm 0.02$ & $1.0^{b}$ & \\
\hline & & Si II* 1533 & - & - & $<12.5$ & - & $\mathrm{nl}$ \\
\hline & & C IV 1548 & 4544.40 & $\sim 25$ & $>14.2$ & $1.0^{a}$ & sat $\&$ bl, adopt $b$ from Si IV \\
\hline & & C IV 1551 & 4551.94 & - & - & - & - \\
\hline & & Mg II 2796 & 8208.39 & $20.8 \pm 1.1$ & $13.31 \pm 0.03$ & $1.0^{b}$ & \\
\hline & & Mg II 2804 & 8229.47 & - & - & - & \\
\hline
\end{tabular}

\footnotetext{
${ }^{a}$ Heavily saturated lines, whose $C_{0}$ equals the observed depth of the line.

${ }^{b}$ Unsaturated doublets, where we solve for $C_{0}$ using equation (3).

${ }^{c}$ Unsaturated single lines, where we adopt $C_{0}=1$ because it is not constrained.

${ }^{d}$ Weak doublets, where we take a conservative approach by setting $C_{0}=1$.
}

to Si IV and, therefore, it seems likely that Si III is broader than Si II because it has contributions from regions of higher ionization.

We apply the same procedure to derive $U$ values and ranges for the other quasars, again assuming solar abundance ratio if ion pairs in the same elements are not available. Table 7 lists the $U$ values and the ions used for each component.

To estimate the total hydrogen column densities, we assume that the measured H I column densities reside primarily with the lower metal ions (see Section 4.5 for more discussion). For example, for component 2 in Q0119-046, we apply an ionization correction $\mathrm{H} \mathrm{I} / \mathrm{H} \approx-3.2$ based on $\log U \sim-1.9$ from $\mathrm{Si} \mathrm{II} / \mathrm{Si}$ III to the observed $N(\mathrm{HI})$ value to derive a total column density of $\log N(\mathrm{H})\left(\mathrm{cm}^{-2}\right)=$ 20.9 in this absorber. For the other quasars with only conservative lower limits on $N(\mathrm{HI})$, we derive conservative lower limits on $N(\mathrm{H})$. These results are also listed in Table 7.

It is important to note that the results for $U$ and $N(H)$ for the other quasars, with only lower limits on $N(\mathrm{HI})$, are based on CLOUDY models where the absorber is optically thin in the ionizing continuum (Section 4.2). If shielding does play a role, then the total column densities would need to be at least as large as Q0119-046, where $\log U \sim-1.9$ and $\log N(\mathrm{H})\left(\mathrm{cm}^{-2}\right)=20.9$ combined to produce significant absorption at the H I Lyman edge. We cannot exclude this possibility for the other quasars, but their absorbers are clearly different from Q0119-046 given their lower densities and larger distances (discussed below).

Another constraint on the ionization and densities comes from the neutral $\mathrm{Mg}$ I $\lambda 2853$ line that appears uniquely in components 2 and 3 of Q0105+061. This ion cannot be shielded behind an H II-H I recombination front because its ionization potential $7.65 \mathrm{eV}$ is well below that of $\mathrm{HI}$ at $13.6 \mathrm{eV}$. Therefore, the $\mathrm{Mg}$ I lines suggest that high densities lead to very low ionization parameters in some portions of these absorbers. Our CLOUDY simulations (Fig. 10) indicate that significant amounts of $\mathrm{Mg}$ I require $\log U<-5$. (The results are the same if we use larger column densities like Q0119-046 because the shielding effects for $\mathrm{Mg}$ I are still negligible.) For component 2 in Q0105+061 with estimated $\log U \approx-3.0$ in the Si II region (top panel of Fig. 10), we estimated an upper limit on the density of $n_{\mathrm{e}} \lesssim 7 \mathrm{~cm}^{-3}$ (Table 7). If the $\mathrm{Mg}$ I line forms at the same radial distance as Si II, then there would need to be regions with densities $\gtrsim 100$ times larger than the Si II gas to support measurable $\mathrm{Mg}$ I.

\subsection{Radial distance}

Here we estimate the radial distance, $R$, of the Si II or C II AAL regions from the quasars using $n_{\mathrm{e}}$ and $U$ in the Si II or C II regions 
Table 5. Individual absorption lines of Q0334-204. See Table 2 for descriptions of the table contents. For the notes, $\mathrm{nl}=\mathrm{no}$ obvious lines, $\mathrm{bl}=\mathrm{blended}$ with neighbouring systems, sat $=$ saturated line, $\mathrm{w}=$ weak line. For $N(\mathrm{H} \mathrm{I})$ lower limit estimates, we adopt $b$ from $\mathrm{C}$ IV, and assume $\tau_{0}>3$ in Ly $\delta 950 \AA$. We use Ly $\delta$ because Ly $\delta$ has smaller oscillator strength than $\operatorname{Ly} \alpha$, Ly $\beta$, and Ly $\gamma$, which results in more accurate estimation of the lower limit.

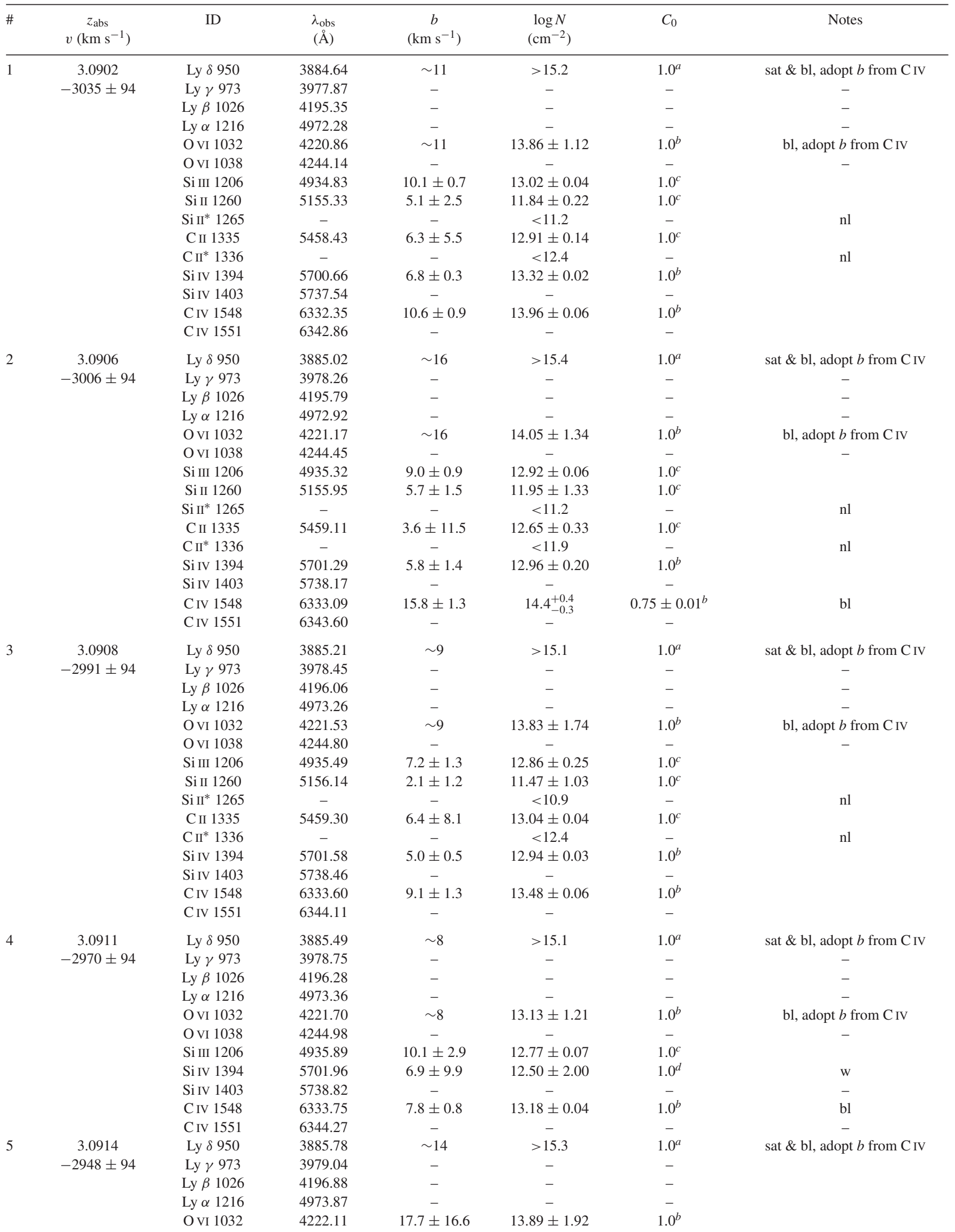


Table 5 - continued

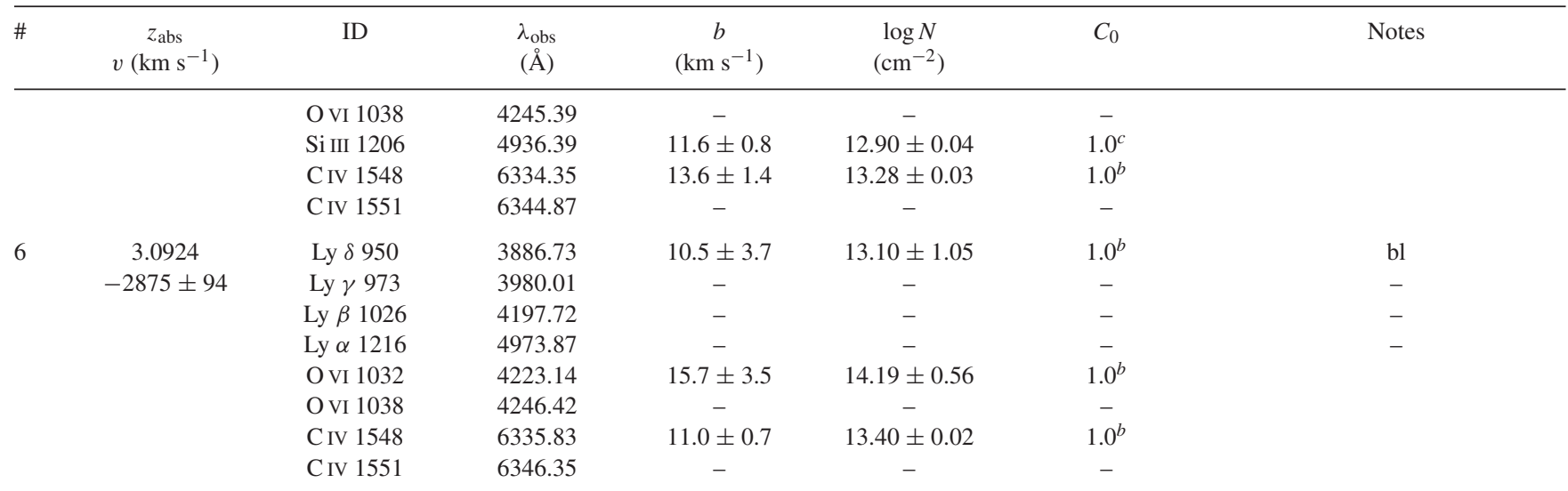

\footnotetext{
${ }^{a}$ Heavily saturated lines, whose $C_{0}$ equals the observed depth of the line.

${ }^{b}$ Unsaturated doublets, where we solve for $C_{0}$ using equation (3).

${ }^{c}$ Unsaturated single lines, where we adopt $C_{0}=1$ because it is not constrained.

${ }^{d}$ Weak doublets, where we take a conservative approach by setting $C_{0}=1$.
}

derived in Sections 4.1 and 4.3, respectively. First we combine our adopted quasar spectrum from Section 4.2 with equations (8) and (9) to derive an expression for the distance,

$$
R=40.8\left(\frac{\nu L_{v}(1500 \AA)}{10^{46} \mathrm{erg} \mathrm{s}^{-1}}\right)^{\frac{1}{2}}\left(\frac{10 \mathrm{~cm}^{-3}}{n_{\mathrm{H}}}\right)^{\frac{1}{2}}\left(\frac{0.01}{U}\right)^{\frac{1}{2}} \mathrm{kpc},
$$

where $v L_{v}(1500 \AA)$ is the monochromatic luminosity at $1500 \AA$, and we assume $n_{\mathrm{e}} \approx n_{\mathrm{H}}$ for an ionized gas. We estimate luminosities for each quasar using the $g$-band photometry from the Sloan Digital Sky Survey (SDSS). This provides a flux at rest wavelengths near $1500 \AA$, which we then extrapolate to $1500 \AA$ using a power law with spectral index $\alpha_{\mathrm{OX}}=-1.7$. We estimate the bolometric luminosity, $L_{\mathrm{Bol}}$, for each quasar via integration of the SED, $\sim 6.76 v L_{v}(1500 \AA$ A) (Table 7). For Q0119-046, the derived density and $\log U \sim-1.9$ appropriate for the $\mathrm{C}$ II and $\mathrm{Si}$ II absorbing region yields a distance of $R \sim 5.7 \mathrm{kpc}$ (see Table 7). The errors listed for this distance derive from the uncertainty in $U$. For the other quasars, we combine the density upper limits with $\log U$ listed in Table 7 to derive conservatively small distance lower limits that range from $R$ $\gtrsim 40$ to $330 \mathrm{kpc}$.

\subsection{Gas metallicity}

We estimate metallicities only for component 2 in Q0119-046 where $N(\mathrm{HI})$ is well measured. [The lower limits on $N(\mathrm{HI})$ in the other quasars lead to large upper limits on the metallicities, well above solar, that do not provide useful constraints.] The metallicity can be written generally as

$$
\left[\frac{\mathrm{M}}{\mathrm{H}}\right]=\log \left(\frac{N\left(\mathrm{M}_{i}\right)}{N(\mathrm{HI})}\right)+\log \left(\frac{f(\mathrm{HI})}{f\left(\mathrm{M}_{i}\right)}\right)+\log \left(\frac{\mathrm{H}}{\mathrm{M}}\right)_{\odot},
$$

where $(\mathrm{H} / \mathrm{M}) \odot$ is the solar abundance ratio of hydrogen to some metal $\mathrm{M}, N(\mathrm{HI})$, and $f(\mathrm{HI})$ are the column density and ionization fraction in $\mathrm{H}$, respectively, and $N\left(\mathrm{M}_{i}\right)$ and $f\left(\mathrm{M}_{i}\right)$ are the column density and ionization fraction in some ion $\mathrm{M}_{i}$ of metal $\mathrm{M}$.

To calculate $[\mathrm{Si} / \mathrm{H}]$ and $[\mathrm{C} / \mathrm{H}]$ for this absorber, we first use $\log U$ $\approx-1.9$ obtained from the Si II and Si III region to determine the ionization fractions $f(\mathrm{C} \mathrm{II}), f(\mathrm{Si}$ II $)$, and $f(\mathrm{HI})$ from our calculation in Fig. 9. Then the measured values of $N(\mathrm{Si}$ II $)$ and $N(\mathrm{C}$ II $)$ combined with $N(\mathrm{HI})$ indicate $[\mathrm{C} / \mathrm{H}] \approx-1.8 \pm 0.1$ and $[\mathrm{Si} / \mathrm{H}] \approx-2.4 \pm 0.2$. From Section 4.3, we know there is a range of ionization for this component. If assuming the high ions $\mathrm{O}$ VI and Ne VIII have similar metallicity as the low ions $\mathrm{Si}$ II and C II, then we use the $\log U \sim 0.9$ from the ratios $\mathrm{O} \mathrm{VI} / \mathrm{Ne} \mathrm{VIII}$, and the corresponding $f(\mathrm{HI}) \sim-6.7$ and $f(\mathrm{O}$ VI $) \sim-1.4$ to predict $N(\mathrm{HI})$. The predicted $\log N(\mathrm{HI})$ is $\sim 15.5$, which is much less than $\sim 17.7$. This is the reason why we assume that the measured $\mathrm{H}$ I column densities reside primarily with the lower metal ions (Section 4.3).

\subsection{Spatial structure and cloud survival}

There is partial covering in roughly half of the components in Q0119-046. This implies that absorbers are not much larger (and probably smaller) than the projected area of the emission regions. The partial covering in C IV and Ly $\alpha$ appears to apply to the broad emission line region (BLR) because (1) these absorption lines sit on top of strong broad emission lines (Sargent et al. 1982), (2) the covering fraction in C IV is only slightly less than Si IV, which does not sit on a strong emission line, and (3) the bottoms of the Ly $\alpha$ troughs are slanted in a way that is consistent with the peak of the broad Ly $\alpha$ emission line partially filling in the troughs. The BLR scaling relationship in Bentz et al. (2013) indicates that the size of the broad emission region is $\sim 1.0 \mathrm{pc}$, and therefore the absorbers have transverse size $\lesssim 1.0 \mathrm{pc}$. We also find evidence for partial covering in O VI, Ne VIII and the higher Lyman lines. Since these features do not sit on strong emission lines, they must partially cover the much smaller continuum source. This requires the absorber sizes that are conservatively less than 0.01 pc (Netzer 1992; Hamann et al. 2011; Hamann et al. 2018).

These small cloud sizes in Q0119-046 are surprising given the derived radial distance of $\sim 5.7 \mathrm{kpc}$, i.e. far for the quasar in the host galaxy. Such clouds are not expected in a normal galactic interstellar medium, but they not unprecedented for distant AAL absorbing regions. For example, Hamann et al. (2001) found partial covering 
Table 6. Individual absorption lines of Q2044-168. See Table 2 for descriptions of the table contents. For the notes, nl $=$ no obvious lines, sat $=$ saturated line, $\mathrm{bl}=$ blended with neighbouring systems, unbl = blended with unrelated lines (e.g. lines in the Lyman forest).

\begin{tabular}{|c|c|c|c|c|c|c|c|}
\hline \# & $\begin{array}{c}z_{\mathrm{abs}} \\
v\left(\mathrm{~km} \mathrm{~s}^{-1}\right)\end{array}$ & ID & $\begin{array}{l}\lambda_{\text {obs }} \\
(\AA)\end{array}$ & $\begin{array}{c}b \\
\left(\mathrm{~km} \mathrm{~s}^{-1}\right)\end{array}$ & $\begin{array}{c}\log N \\
\left(\mathrm{~cm}^{-2}\right)\end{array}$ & $C_{0}$ & Notes \\
\hline 1 & $\begin{array}{c}1.9183 \\
-2113 \pm 102\end{array}$ & $\begin{array}{l}\text { Si III } 1206 \\
\text { Ly } \alpha 1216 \\
\text { C II } 1335 \\
\text { C II* } 1336 \\
\text { Si IV } 1394 \\
\text { Si IV } 1403 \\
\text { C IV } 1548 \\
\text { C IV } 1551\end{array}$ & $\begin{array}{c}3520.94 \\
3547.58 \\
3894.54 \\
- \\
4067.42 \\
4093.71 \\
4518.09 \\
4525.59\end{array}$ & $\begin{array}{c}8.6 \pm 0.7 \\
\sim 17 \\
10.2 \pm 4.4 \\
- \\
13.6 \pm 0.6 \\
- \\
16.5 \pm 0.6 \\
-\end{array}$ & $\begin{array}{c}12.51 \pm 0.03 \\
>13.8 \\
13.0_{-0.5}^{+0.1} \\
<12.7 \\
12.89 \pm 0.01 \\
- \\
14.35 \pm 0.04 \\
-\end{array}$ & $\begin{array}{l}1.0^{c} \\
1.0^{a} \\
1.0^{c} \\
- \\
1.0^{b} \\
- \\
1.0^{b} \\
-\end{array}$ & sat $\&$ bl, adopt $b$ from C IV \\
\hline 2 & $\begin{array}{c}1.9190 \\
-2042 \pm 102\end{array}$ & $\begin{array}{c}\text { Si III } 1206 \\
\text { Ly } \alpha 1216 \\
\text { N V } 1239 \\
\text { N V } 1243 \\
\text { Si II } 1260 \\
\text { Si II } 1265 \\
\text { C II } 1335 \\
\text { C II* } 1336 \\
\text { Si IV } 1394 \\
\text { Si IV } 1403 \\
\text { C IV } 1548 \\
\text { C IV } 1551 \\
\text { Mg II } 2796 \\
\text { Mg II } 2804\end{array}$ & $\begin{array}{c}3521.77 \\
3548.51 \\
3616.07 \\
3627.70 \\
3679.17 \\
- \\
3895.49 \\
- \\
4068.34 \\
4094.66 \\
4519.15 \\
4526.65 \\
8162.36 \\
8183.31\end{array}$ & $\begin{aligned} 9.0 & \pm 0.5 \\
\sim & 16 \\
12.2 & \pm 1.7 \\
- & \\
8.5 & \pm 8.1 \\
- & \\
8.2 & \pm 0.4 \\
- & \\
9.0 & \pm 0.3 \\
- & \\
15.8 & \pm 0.7 \\
- & \\
15.2 & \pm 5.9 \\
- & \end{aligned}$ & $\begin{array}{c}13.01 \pm 0.04 \\
>13.8 \\
13.54 \pm 0.06 \\
- \\
12.26 \pm 0.27 \\
<11.2 \\
13.45 \pm 0.02 \\
<12.3 \\
13.34 \pm 0.02 \\
- \\
14.44 \pm 0.04 \\
- \\
<12.49 \\
-\end{array}$ & $\begin{array}{c}1.0^{c} \\
1.0^{a} \\
1.0^{b} \\
- \\
1.0^{c} \\
- \\
1.0^{c} \\
- \\
1.0^{b} \\
- \\
1.0^{b} \\
- \\
1.0^{b} \\
-\end{array}$ & $\begin{array}{c}\mathrm{nl} \\
\mathrm{nl} \\
\\
\mathrm{unbl} \\
-\end{array}$ \\
\hline 3 & $\begin{array}{c}1.9198 \\
-1960 \pm 102\end{array}$ & $\begin{array}{l}\text { Si III } 1206 \\
\text { Ly } \alpha 1216 \\
\text { C II } 1335 \\
\text { C II* } 1336 \\
\text { Si IV } 1394 \\
\text { Si IV } 1403 \\
\text { C IV } 1548 \\
\text { C IV } 1551\end{array}$ & $\begin{array}{c}3522.76 \\
3549.45 \\
3896.55 \\
- \\
4069.43 \\
4095.75 \\
4520.42 \\
4527.92\end{array}$ & $\begin{aligned} & 21.5 \pm 1.0 \\
& \sim 8 \\
& 8.5 \pm 1.7 \\
&- \\
& 5.0 \pm 0.7 \\
&- \\
& 8.1 \pm 5.7 \\
&-\end{aligned}$ & $\begin{array}{c}13.10 \pm 0.04 \\
>13.5 \\
12.76 \pm 0.01 \\
<12.1 \\
12.22 \pm 0.03 \\
- \\
13.33 \pm 0.04 \\
-\end{array}$ & $\begin{array}{l}1.0^{c} \\
1.0^{a} \\
1.0^{c} \\
- \\
1.0^{b} \\
- \\
1.0^{b} \\
-\end{array}$ & $\begin{array}{c}\mathrm{bl} \\
\text { sat } \& \mathrm{bl} \text {, adopt } b \text { from C IV } \\
\mathrm{nl}\end{array}$ \\
\hline 5 & $\begin{array}{c}1.9206 \\
-1878 \pm 102\end{array}$ & $\begin{array}{l}\text { Si III } 1206 \\
\text { Ly } \alpha 1216 \\
\text { C II } 1335 \\
\text { C II* } 1336 \\
\text { Si IV } 1394 \\
\text { Si IV } 1403 \\
\text { C IV } 1548 \\
\text { C IV } 1551\end{array}$ & $\begin{array}{c}3523.66 \\
3550.44 \\
3897.59 \\
- \\
4070.56 \\
4096.89 \\
4521.65 \\
4529.16\end{array}$ & $\begin{array}{c}9.0 \pm 0.9 \\
\sim 11 \\
14.8 \pm 2.1 \\
- \\
6.7 \pm 0.6 \\
- \\
10.6 \pm 0.5 \\
-\end{array}$ & $\begin{array}{c}12.30 \pm 0.04 \\
>13.6 \\
13.0_{-0.3}^{+0.2} \\
<12.5 \\
12.39 \pm 0.05 \\
- \\
13.78 \pm 0.04 \\
-\end{array}$ & $\begin{array}{l}1.0^{c} \\
1.0^{a} \\
1.0^{c} \\
- \\
1.0^{b} \\
- \\
1.0^{b} \\
-\end{array}$ & $\begin{array}{c}\text { sat } \& \text { bl, adopt } b \text { from C IV } \\
\mathrm{nl}\end{array}$ \\
\hline
\end{tabular}

${ }^{a}$ Heavily saturated lines, whose $C_{0}$ equals the observed depth of the line.

${ }^{b}$ Unsaturated doublets, where we solve for $C_{0}$ using equation (3).

${ }^{c}$ Unsaturated single lines, where we adopt $C_{0}=1$ because it is not constrained.

of the continuum source in an AAL absorber $\sim 28 \mathrm{kpc}$ from the central quasar. If the clouds are not confined by an external pressure, they will dissipate in roughly a sound crossing time (Hamann et al. 2001; Finn et al. 2014) given by

$t_{\mathrm{sc}}=\frac{l}{c_{\mathrm{s}}}$ where $c_{\mathrm{S}}$ is the sound speed and $l$ is the characteristic cloud size (Hamann et al. 2001; Schaye 2001; Finn et al. 2014). For a nominal temperature of $10^{4} \mathrm{~K}$ and $l \lesssim 0.01$ pc or more conservatively $l \lesssim$ $1 \mathrm{pc}$, the cloud survival times are $\lesssim 700$ or $\lesssim 70000 \mathrm{yr}$, respectively. The gas speeds in the various AAL systems in Q0119-046 are overall $\lesssim 1200 \mathrm{~s}^{-1}$, which means that the cloud survival times are 


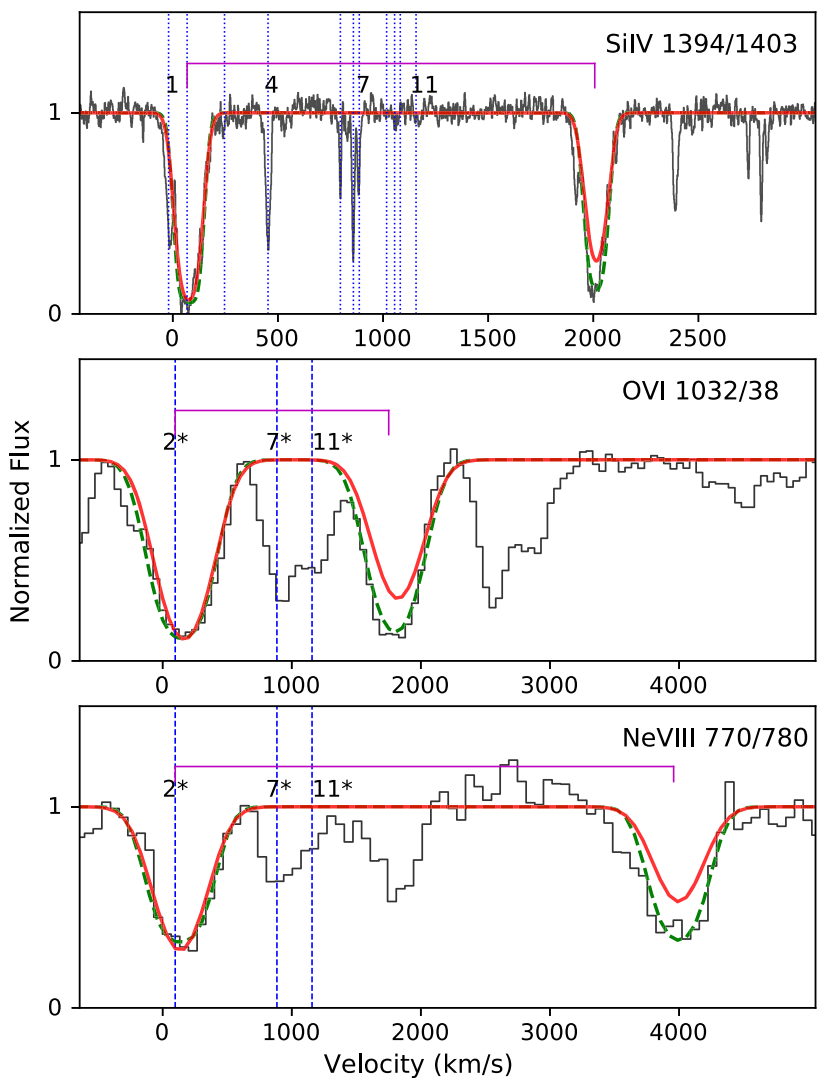

Figure 7. Observed spectra of Si IV $\lambda 1394,1403$ (from Keck), O VI $\lambda 1032$, 1038, and Ne VIII $\lambda 770,780$ (from HST) of Q0119-046 (shown in black in each panel) are compared to predicted lines (component 2 or $2^{*}$ ) based on Si IV $\lambda 1394$, O VI $\lambda 1032$, and Ne VIII $\lambda 770$ assuming $C_{0}=1$ (red solid lines). The green dash lines in each panel show the final fitting results when $C_{0}<$ 1. The blue dash lines are components, and the brackets show the doublets. The velocities pertain to the short-wavelength lines in the doublets.

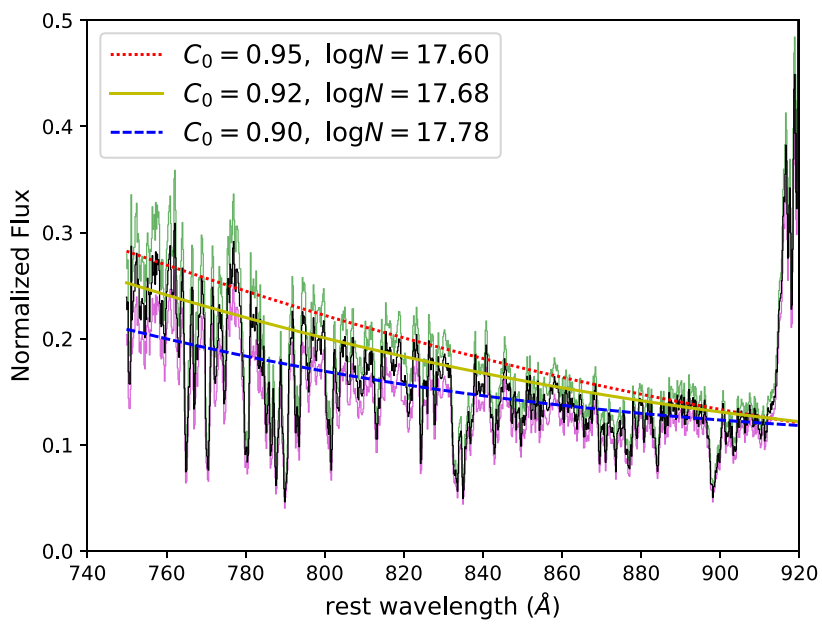

Figure 8. Observed spectrum normalized in three different ways using the continuum fits from Section 2.2.1 (Fig. 1). Column densities values change with different covering fractions by fitting the Lyman limit. The green and mauve spectra are the upper and lower limits of the normalized spectra, respectively. And the black spectrum shows the best normalized spectrum. The dotted red and dashed dark blue lines are the upper and lower limits of fits considering uncertainties of normalization. The range of $C_{0}$ is 0.90 $(\log N=17.78)$ to $0.95(\log N=17.60)$. The best fit is $C_{0}=0.92$ and $\log N=17.68$, shown as the solid yellow line. much shorter than any reasonable flow time we might assign to these absorbers. It therefore seems likely that the clouds were created in situ, at or near their observed location $\sim 5.7 \mathrm{kpc}$ from the quasar (see Section 5 for more discussion).

\section{DISCUSSION}

The information derived in Sections 3 and 4 provides valuable constraints on the nature and origins of the AALs in our quasar sample. The results might have general relevance to high-redshift quasar environments, but it is important to keep in mind that the sample is biased. The quasars were selected to have rare low-ionization lines ( $\mathrm{Si}$ II and/or $\mathrm{C}$ II) useful for density and location constraints. More common types of AALs, with only higher ionization lines, are not included in our sample. The full range of ions detected in our study, from Mg I, Mg II, Si II, C II, up to C IV, N v, and in some cases $\mathrm{O}$ VI and Ne VIII, often require a range of ionization parameters in the AAL gas. If the diverse lines in each system form at roughly the same location, then the absorbing regions must span a range of densities. The most extreme case is component 2 in Q0119-046, where ions ranging from Si II to Ne VIII indicate a factor of $\sim 630$ range in densities (Section 4.3). This result is similar to several other quasars where measured Ne VIII AALs also indicate a range of ionizations and densities (Hamann et al. 1995; Petitjean \& Srianand 1999; Hamann, Netzer \& Shields 2000; Arav et al. 2013; Muzahid et al. 2013; Finn et al. 2014)

In our study, Q0119-046 has a unique dataset because we combine high-resolution spectra from Keck-HIRES with spectra from HST-FOS that reach wavelengths down to $\sim 750 \AA$ in the quasar rest frame. These data provide the best measurements and different results than the other three AAL quasars in our sample. Thus we discuss Q0119-046 separately below.

\subsection{Q0119-046}

The AALs in Q0119-046 are clearly intrinsic to the quasar environment based on multiple components with partial covering of the background light source and high densities of $n_{\mathrm{H}} \sim 2500 \mathrm{~cm}^{-3}$ that lead to a derived distance of $R \sim 5.7 \mathrm{kpc}$. These specific density and location results are similar to the absorbers studied by Dunn et al. (2010), Arav et al. (2013), and Finn et al. (2014), which have $n_{\mathrm{H}} \sim 1000-6000 \mathrm{~cm}^{-3}$ and $R \sim 2-6 \mathrm{kpc}$. The partial covering we find in Q0119-046 additionally implies that the absorber is composed of small clouds with characteristic sizes $\lesssim 1 \mathrm{pc}$ and possibly $\lesssim 0.01 \mathrm{pc}$ (if the partial covering applies to the continuum source, Section 4.6). Our results overall are consistent with the previous study of Q0119-046 by Sargent et al. (1982) that obtained only a lower limit on the density from $\mathrm{C} \mathrm{II}^{*} / \mathrm{C}$ II and an upper limit on the distance of $<60 \mathrm{kpc}$. The AALs in Q0119-046 join a growing number of well-measured systems in other quasars where density indicators place the absorbers within the quasar environments but at large distances of a few to a few hundred kpc, and where partial covering of the quasar emission source can require small cloud sizes at these distances (see also Tripp et al. 1996; Barlow \& Sargent 1997; Srianand \& Petitjean 2000; Hamann et al. 2001; Gabel, Arav \& Kim 2006; Arav et al. 2008)

The radial distance $R \sim 5.7 \mathrm{kpc}$ of the absorber in Q0119-046 is an interesting location where a quasar-driven wind might be interacting with interstellar gas in the extended host galaxy. The derived metallicities in the range $\sim 0.004$ to $\sim 0.016 Z_{o}$ are substantially smaller than expected for an outflow originating in the galactic 
Table 7. Parameters of some absorbers. Columns show quasar name, bolometric luminosity in $10^{47} \mathrm{ergs} \mathrm{s}^{-1}$, component, velocity shifts in km s ${ }^{-1}$, column density ratio of $\mathrm{C} \mathrm{II}^{*}$ and $\mathrm{C}$ II, column density ratio of Si II* and Si II, electron density, ionization parameter, total hydrogen column density, and radial distance in kpc.

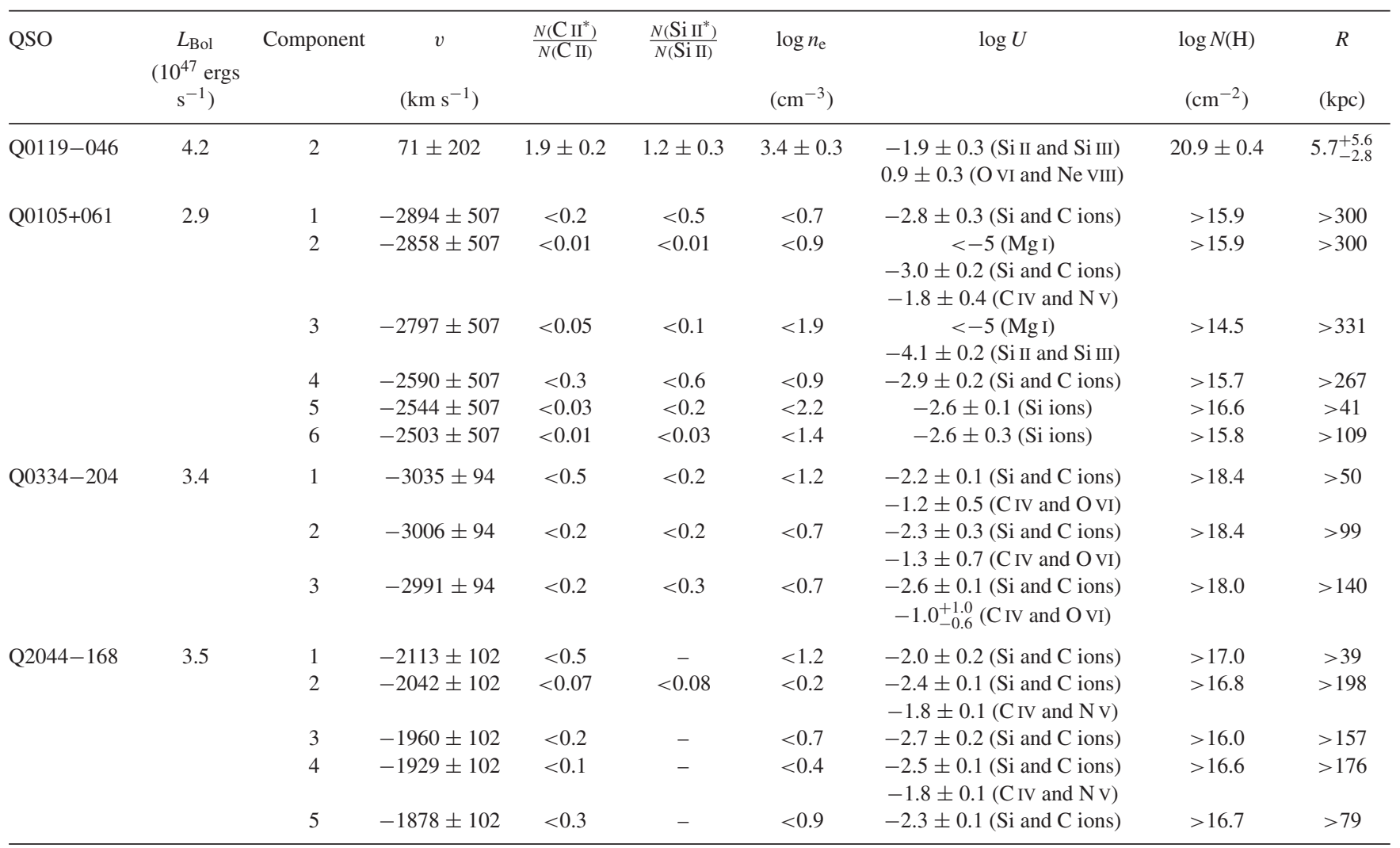

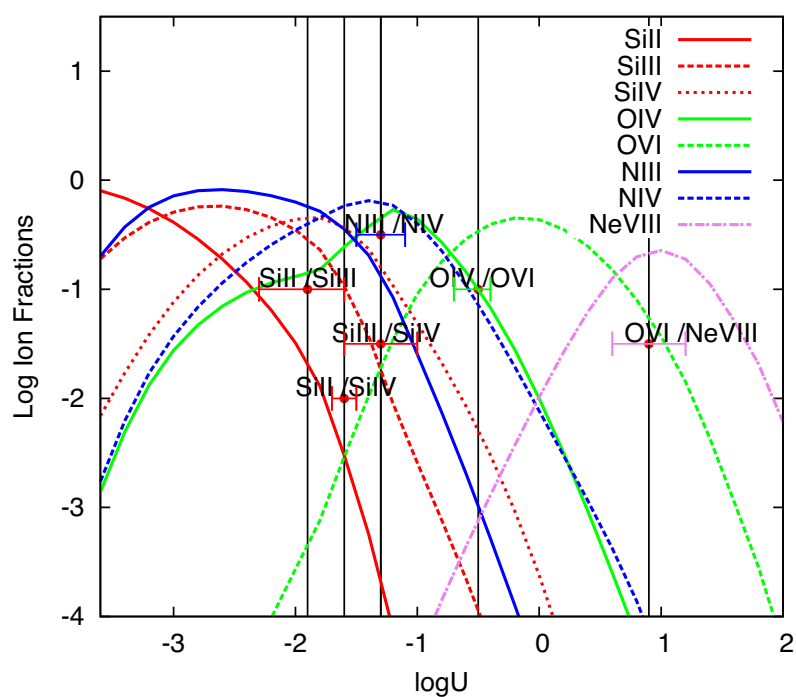

Figure 9. Theoretical ionization fractions, $f\left(\mathrm{M}_{i}\right)$, for selected stages of the elements $\mathrm{Si}, \mathrm{N}, \mathrm{O}$, and Ne plotted against different ionization parameters $\log U$ for component 2 in Q0119-046. The black vertical lines with error bars are the best estimations of $U$ for each ion pair.

nuclear regions near the quasar, where solar or higher metallicities should be present (Hamann \& Ferland 1999; Arav et al. 2001; Hamann et al. 2002; Dietrich et al. 2003; Warner, Hamann \& Dietrich 2004; Gabel et al. 2005; Nagao, Marconi \& Maiolino 2006; Simon \& Hamann 2010). If this AAL complex stems from a quasar- driven outflow, then the low metallicities might imply that the outflow gas is mixed with ambient interstellar gas in the galaxy. The multicomponent nature of the AAL complex might identify interstellar clouds that have been shredded and dispersed by an unseen high-speed quasar-driven outflow, as described in some recent theoretical models (Hopkins \& Elvis 2010; Faucher-Giguère \& Kereš 2011; Faucher-Giguère, Quataert \& Murray 2012). This interpretation is appealing because it provides a natural mechanism for creating small absorption-line clouds in situ, and thus avoiding the problem of their short survival times (see refs above, also Section 4.6).

However, another possibility is that the AAL absorbers in Q0119-046 represent infalling metal-poor gas from the intergalactic medium (IGM). The measured line velocities favour this infall interpretation. Components 1 and 2 have velocity shifts consistent with zero, while the other systems span a range of positive velocities from $\sim 240$ to $\sim 1150 \mathrm{~s}^{-1}$ (Table 2 and Section 2.1). In this infall scenario, small absorption-line clouds could be created in situ as condensations in larger reservoir of cold-mode accreting gas (Kerě̌ et al. 2009; Fumagalli et al. 2011; Hafen et al. 2017). McCourt et al. (2016) describe theoretically that clouds of optically thin, pressureconfined gas are inclined to fragment as they cool, reaching very small size scales of $\sim 0.1 \mathrm{pc}$.

Accurate AAL velocity shifts are critical for this analysis. We adopt a redshift for Q0119-046 from Steidel \& Sargent (1991) based on the $\mathrm{Mg}$ II broad emission line, with estimated measurement uncertainties of $\lesssim 100 \mathrm{~s}^{-1}$ (Section 2.1). Another possible source of uncertainty is the offset of the $\mathrm{Mg}$ II line from the true systemic rest frame of the quasar environment. Studies of large quasar samples 

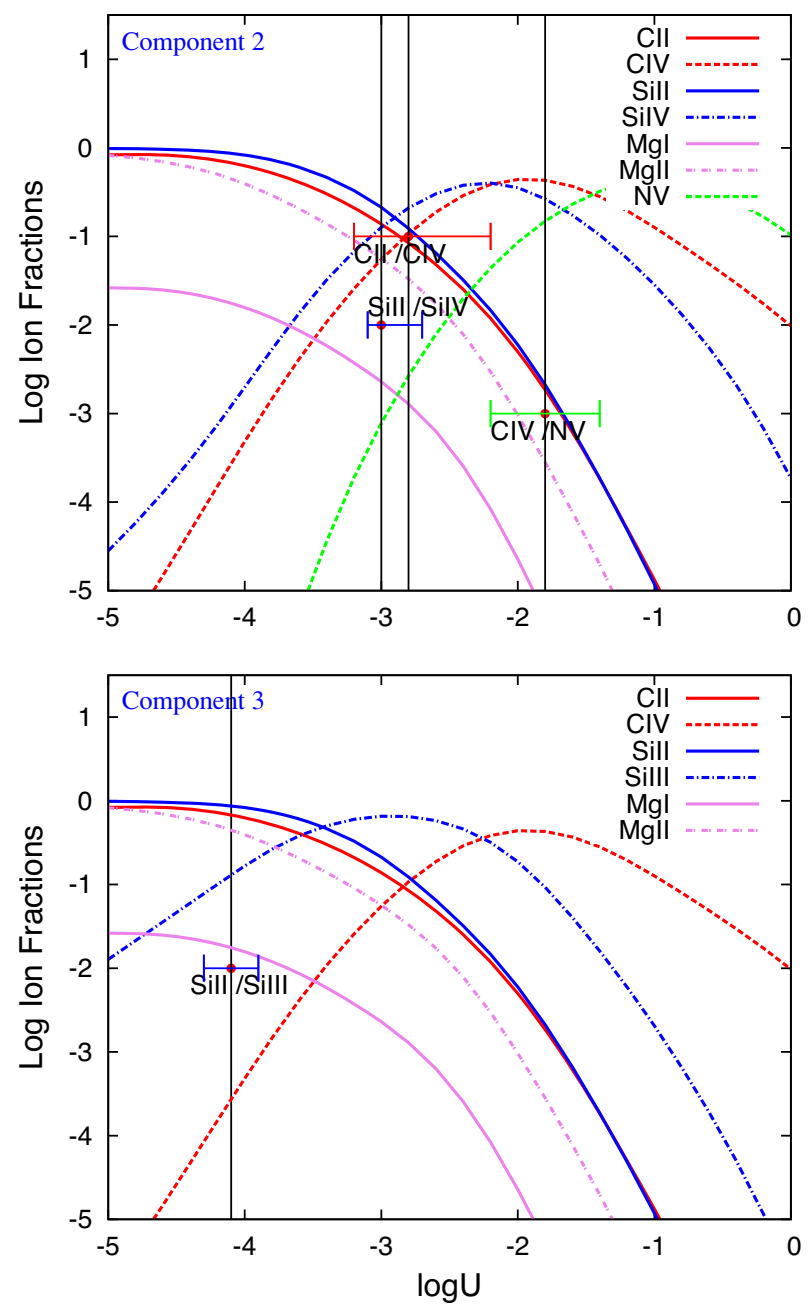

Figure 10. Theoretical ionization fractions, $f\left(\mathrm{M}_{i}\right)$, for selected stages of the elements $\mathrm{Si}, \mathrm{C}, \mathrm{Mg}$, and $\mathrm{N}$ plotted against different ionization parameters $\log U$ for components 2 and 3 in Q0105+061. The black vertical lines with error bars are the best estimations of $U$ for each ion pair.

indicate that the mean offset of the $\mathrm{Mg}$ II emission line relative to [O III] $\lambda 5007$ corresponds to a slight blueshift of $\sim 100 \mathrm{~s}^{-1}$ with $1 \sigma$ scatter of $\sim 270 \mathrm{~s}^{-1}$ (Richards et al. 2002; Shen et al. 2007, 2016). If the [OIII] line is a better redshift indicator, the probability for a random $\mathrm{Mg}$ II blueshifted matching our measured shift of $\sim 1150 \mathrm{~s}^{-1}$ is less than 0.005 per cent $(\sim 4 \sigma)$. We conclude that at least some of the AAL components in Q0119-046 form in gas that is infalling towards the quasar.

Infalling gas (e.g. cold mode accretion) from the IGM is believed to be important during the early stages of galaxy formation to build mass, trigger star formation, and fuel the central black hole (Katz et al. 2003; Kereš et al. 2009, 2012). It is likely that infall and outflow occur together if cold-mode accretion is involved in triggering the starbursts and quasars that also drive feedback (Costa et al. 2014; Nelson et al. 2015; Suresh et al. 2015). Recent observations show that massive gas reservoirs are indeed present around highredshift quasars, and that they are more extended and more massive around quasar hosts than similar inactive (non-quasar) galaxies (e.g. Prochaska et al. 2014; Johnson et al. 2015; Martin et al. 2015, 2016; Borisova et al. 2016; Bouché et al. 2016; Ho et al. 2017). The nature of these gas reservoirs is poorly understood, but they are consistent with enhanced infall/cold-mode accretion from the IGM during an
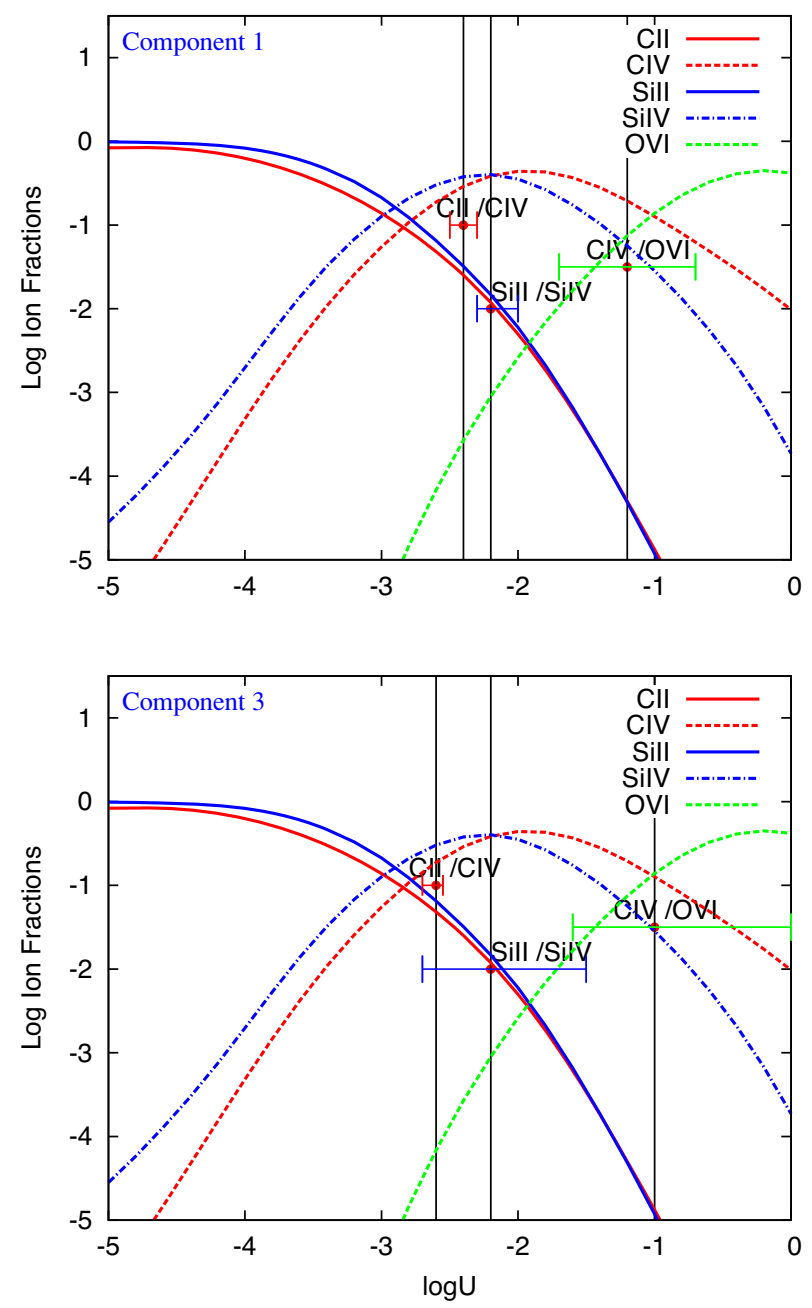

Figure 11. Theoretical ionization fractions, $f\left(\mathrm{M}_{i}\right)$, for selected stages of the elements $\mathrm{Si}, \mathrm{C}$, and $\mathrm{O}$ plotted against different ionization parameters $\log U$ for components 1 and 3 in Q0334-204. The black vertical lines with error bars are the best estimations of $U$ for each ion pair.

early active stage of massive galaxy evolution when there is ongoing quasar activity. Quasar AALs can be valuable tracers of infall because they measure the gas speeds and physical conditions along radial lines of sight into galactic nuclei. The AALs in Q0119-046 might provide direct observational evidence for infall related to the assembly of a massive galaxy at redshift $z \sim 1.96$.

Compared to the work by Sargent et al. (1982), we obtain more accurate results, especially $N(\mathrm{HI})$ and $N(\mathrm{H})$, based on the highresolution Keck spectrum and more lines including the Lyman series and the Lyman limit from the $H S T$ spectrum. We estimate $n_{\mathrm{H}}=$ $10^{3.4 \pm 0.3} \mathrm{~cm}^{-3}$ via the strength ratio of $\mathrm{Si}$ II*/Si II. While they constrained $n(\mathrm{H})>100 \mathrm{~cm}^{-3}$ based on the existence of excited state $\mathrm{C}$ II $^{*}$. The radial distance we obtain is $\sim 5.7 \mathrm{kpc}$ because of our accurate $n_{\mathrm{H}}$ estimates. While they estimated its radial distance to be less than $60 \mathrm{kpc}$ by assuming $n_{\mathrm{H}}>100 \mathrm{~cm}^{-3}$ and $N(\mathrm{H})=10^{20} \mathrm{~cm}^{-2}$. We find that $\sim 50$ percent of the absorbers show partial covering cases based on the lines CIV, Si IV, O VI, Ne VIII, Lyman series, and the Lyman limit. This requires absorber size scales less than $0.01 \mathrm{pc}$. While they did not find the partial covering cases. We find that the gas clouds are metal-poor and infalling at velocities from 0 to $1100 \mathrm{~s}^{-1}$ by using the emission-line redshift obtained from $\mathrm{Mg}$ II. While they use the emission line C IV to obtain the systemic red- 

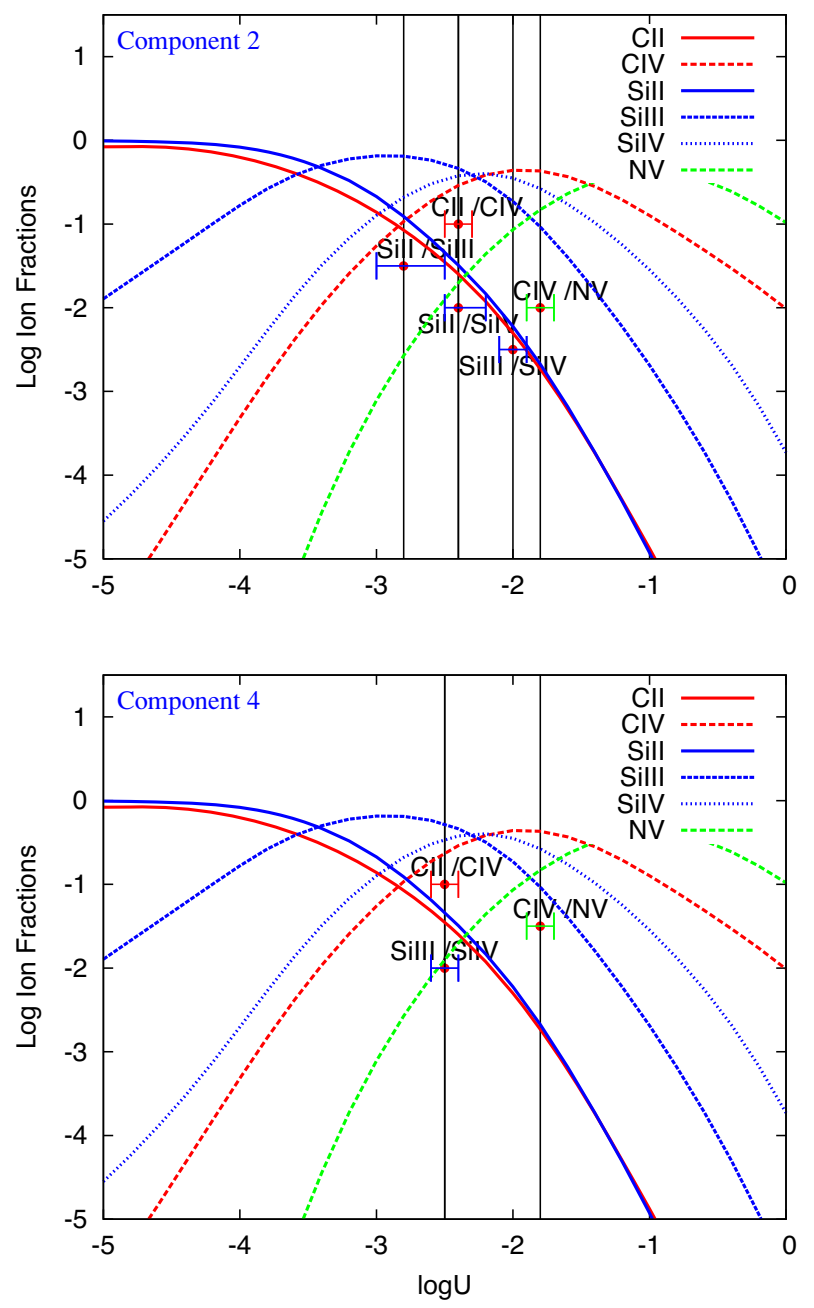

Figure 12. Theoretical ionization fractions, $f\left(\mathrm{M}_{i}\right)$, for selected stages of the elements $\mathrm{Si}, \mathrm{C}$, and $\mathrm{N}$ plotted against different ionization parameters $\log U$ for components 2 and 4 in Q2044-168. The black vertical lines with error bars are the best estimations of $U$ for each ion pair.

shift, which leads to a smaller emission-line redshift than the real one, and thus they obtain a very large infalling speed of $2800 \mathrm{~s}^{-1}$ for the absorber at $z_{\mathrm{abs}}=1.9644$. They guessed that the absorbers are analogous to the NGC 1275 high-velocity filaments (Kent \& Sargent 1979). From our results, we conclude, because of these metal-poor, tiny $(<0.01 \mathrm{pc})$ absorbers and their short survival time $(<700 \mathrm{yr})$, they are created in situ, probably as condensations in cold-mode accreting gas or shredded IGM clouds that are dispersed by an unseen high-speed quasar-driven outflow. In addition, there seems to be no variation between the spectra of the two periods by visual comparisons, if the resolution difference is considered. The lack of variability is consistent with the absorber residing at kpc distances.

\subsection{Q0105+061, Q0334-204, and Q2044-168}

The AALs in the other three quasars in our sample, Q0105+061, Q0334-204, and Q2044-168, have velocity blueshifts consistent with outflows at speeds of -1900 to $-3000 \mathrm{~s}^{-1}$. These systems do not exhibit partial covering of the background light source, and the upper limits we derive on their densities, $\lesssim 150-15 \mathrm{~cm}^{-3}$, place the absorbers at large distances from the quasars, namely, $\gtrsim 40$ to $\gtrsim 330 \mathrm{kpc}$. Thus the relationship of the AAL systems to the quasars is not known. It is possible that they form in intervening gas that is not physically related to the quasars, such as galactic haloes in the same galaxy cluster or group as the quasar host galaxies. However, previous statistical studies of large quasar samples indicate that $\sim 80$ per cent of $\mathrm{C}$ IV AALs with rest equivalent width $\mathrm{REW} \geq 0.3$ $\AA$ at these velocity shifts form in quasar-driven outflows (Misawa et al. 2007a; Nestor et al. 2008; Wild et al. 2008; Simon \& Hamann 2010).

If the AALs we measure do identify quasar outflows, then their speeds and minimum radial distances yield estimates of the minimum outflow masses and kinetic energies. We estimate the total masses assuming the absorbers are part of a spherical shell at distance $R$ from the central quasar, namely

$M=4 \pi \mu m_{\mathrm{p}} Q R^{2} N(\mathrm{H})$,

where $Q$ is the global covering factor of the absorber as seen from the central quasar, $m_{\mathrm{p}}$ is the mass of a proton, and $\mu \sim 1.4$ is the mean molecular weight per proton in an ionized plasma with solar abundances (Hamann 2000; Hamann et al. 2001; Dunn et al. 2010). A rough estimate of $Q$ is the incidence of associated absorbers generally in quasar spectra. Previous studies show that the incidence of AALs in radio-quiet quasars is around 20 per cent (Nestor et al. 2008). Therefore, we adopt $Q=0.2$ to derive minimum total masses of $M \gtrsim(0.1-7.0) \times 10^{7} \mathrm{M}_{\odot}$ for the AAL absorbers in the three quasars. The corresponding minimum kinetic energies, given by $K=M v^{2} / 2$ are quite small, in the range $\sim(2.5-100) \times 10^{48} \mathrm{ergs}$ $\mathrm{s}^{-1}$. Dividing these energies by a characteristic flow time, $\Delta t \sim R / \mathrm{v}$, yields kinetic energy rates, $\dot{K}$, that we compare to the bolometric luminosities (Section 4.4 and Table 7 ) to derive minimum ratios $\dot{K} / L_{\mathrm{Bol}} \gtrsim 10^{-7}-10^{-5}$. The lower limits on these ratios are too small to be important for feedback to the host galaxies, where $\dot{K} / L_{\mathrm{Bol}} \gtrsim$ 0.05-0.005 is believed to be required (Scannapieco \& Oh 2004; Hopkins \& Elvis 2010). However, these estimates are based on conservative lower limits on $N(H)$, which are 2-4 dex smaller than our reliable $N(H)$ measurement for component 2 in Q0119-046.

We conclude that the AAL systems in these three quasars are likely to form in quasar-driven outflows, but their energies and potential for feedback to the host galaxies cannot be determined from existing data.

\section{SUMMARY}

We discuss rest-frame UV spectra of four redshift 2-3 quasars, Q0119-046, Q0105+061, Q0334-204, and Q2044-168, selected to have low-ionization AALs in Si II and/or C II that are valuable to estimate electron densities and radial distances from the quasars. The data include high-resolution spectra obtained with Keck-HIRES or VLT-UVES that we combine, for Q0119-046 only, with an HST-FOS spectrum that reaches down to $\sim 750 \AA$ in the quasar rest frame. Our analysis of Q0119-046 builds upon previous work by Sargent et al. (1982), but with higher quality data and wavelength coverage that measures below the H I Lyman limit. The other three quasars were not previously studied. We fit every detected AAL to measure the gas kinematics, column densities, and line-of-sight covering fractions, and we estimate ionizations, total column densities, and radial distances using CLOUDY photoionization models.

Our main results for Q0119-046 are the following:

(1) The AALs in Q0119-046 identify a complex absorbing structure with at least 11 distinct velocity components. The velocity 
shifts of these components, ranging from $\sim 0$ to roughly $+1150 \mathrm{~s}^{-1}$ (relative to the $\mathrm{Mg}$ II emission line), are indicative of infall towards the quasar with estimated uncertainties of $\lesssim 200 \mathrm{~s}^{-1}$.

(2) The electron density implied by the $\mathrm{Si} \mathrm{II}^{*} / \mathrm{Si}$ II line ratios in component 2, $n_{\mathrm{e}} \sim 2500 \mathrm{~cm}^{-3}$ (Section 4.1), indicates that this absorber resides at a distance $\sim 5.7 \mathrm{kpc}$ from the quasar (Section 4.4 ).

(3) The wide range of ions detected in these AALs, from Si II up to Ne VIII, cannot be attributed to radiative shielding effects inside the absorber. It requires a range of densities from $\sim 2500 \mathrm{~cm}^{-3}$ in the Si II region down to $\sim 4 \mathrm{~cm}^{-3}$ in the Ne VIII gas if the lines all form at roughly the same radial distance (Sections 4.3 and 5.1).

(4) The metallicities in AAL component 2 of Q0119-046 are in the range $\sim 0.004$ to $\sim 0.016$ times solar (Section 4.5 ).

(5) Roughly half of the AAL components in Q0119-046 partially cover the background emission source (Section 4.6). This implies that the absorbers are composed of small clouds with characteristic sizes $\lesssim 1 \mathrm{pc}$ and possibly $\lesssim 0.01 \mathrm{pc}$ (based on O VI, Ne VIII, and the higher Lyman lines that partially cover the quasar continuum source).

(6) These tiny AAL clouds will have short survival times of $\lesssim 700$ or $\lesssim 70000 \mathrm{yr}$ if they are not confined by an external pressure. At the derived distance of $\sim 5.7 \mathrm{kpc}$, the cloud survival times are much less than a flow time, suggesting that the clouds are created in situ, at their observed location.

(7) These results for Q0119-046 are consistent with models of galactic interstellar clouds being shredded and dispersed by a quasar-driven wind. However, the evidence for infall in the line shifts strongly favours an interpretation of this AAL complex as a series of condensations (spanning a factor of $\sim 630$ in density) embedded in a medium that is cold-mode accreting from the intergalactic medium (Section 5.1).

The other three quasars in our sample, Q0105+061, Q0334-204, Q2044-168, have AAL properties similar to each other but different from Q0119-046. Our main results for those quasars are the following:

(1) Non-detections of the excited-state $\mathrm{C}$ II $^{*}$ and $\mathrm{Si}$ II* lines yield upper limits of electron densities from $n_{\mathrm{e}} \lesssim 150$ to $\lesssim 15 \mathrm{~cm}^{-3}$ and lower limits on the radial distances from $R \gtrsim 40$ to $\gtrsim 330 \mathrm{kpc}$ (Sections 4.1 and 4.4).

(2) Most of the components in these AAL systems exhibit a range of ionizations, including the neutral $\mathrm{Mg}$ I $\lambda 2853$ line in Q0105+061. These ranges in ionization again require a range of densities within individual velocity components if the lines all form in roughly the same location (Section 4.3).

(3) There is no evidence for partial covering in any of these AAL systems. Deep saturated lines in some of the components clearly indicate covering fractions of unity.

(4) The AAL velocity shifts are indicative of outflows at speeds of $\sim 1900$ to $\sim 3000 \mathrm{~s}^{-1}$. Previous studies of large quasar samples indicate that $\sim 80$ percent of strong AALs in this velocity range do form in quasar outflows. These AALs might represent highly extended quasar-driven outflows, although the physical relationship of these particular AALs to the quasars cannot be determined (Section 5.2).

\section{ACKNOWLEDGEMENTS}

We thank the anonymous referee for useful comments and suggestions. This work was supported by University of California, Riverside.

\section{REFERENCES}

Anderson S. F., Weymann R. J., Foltz C. B., Chaffee F. H., Jr, 1987, AJ, 94, 278

Arav N., Borguet B., Chamberlain C., Edmonds D., Danforth C., 2013, MNRAS, 436, 3286

Arav N., Kaastra J., Kriss G. A., Korista K. T., Gabel J., Proga D., 2005, ApJ, 620, 665

Arav N., Korista K. T., de Kool M., 2002, ApJ, 566, 699

Arav N., Moe M., Costantini E., Korista K. T., Benn C., Ellison S., 2008, ApJ, 681, 954

Arav N. et al., 2001, ApJ, 561, 118

Bahcall J. N., Wolf R. A., 1968, ApJ, 152, 701

Barlow T. A., Hamann F., Sargent W. L. W., 1997, in Arav N., Shlosman I., Weymann R. J., eds, ASP Conf. Ser. Vol. 128, Mass Ejection from Active Galactic Nuclei. Astron. Soc. Pac., San Francisco, p. 13

Barlow T. A., Sargent W. L. W., 1997, AJ, 113, 136

Baskin A., Laor A., Stern J., 2014, MNRAS, 445, 3025

Bentz M. C. et al., 2013, ApJ, 767, 149

Borisova E., Lilly S. J., Cantalupo S., Prochaska J. X., Rakic O., Worseck G., 2016, ApJ, 830, 120

Bouché N. et al., 2016, ApJ, 820, 121

Chhetri R., Ekers R. D., Jones P. A., Ricci R., 2013, MNRAS, 434, 956

Cicone C. et al., 2014, A\&A, 562, A21

Costa T., Sijacki D., Haehnelt M. G., 2014, MNRAS, 444, 2355

Debuhr J., Quataert E., Ma C.-P., 2012, MNRAS, 420, 2221

de Kool M., Korista K. T., Arav N., 2002, ApJ, 580, 54

Dietrich M., Hamann F., Shields J. C., Constantin A., Heidt J., Jäger K., Vestergaard M., Wagner S. J., 2003, ApJ, 589, 722

Di Matteo T., Springel V., Hernquist L., 2005, Nature, 433, 604

Dunn J. P. et al., 2010, ApJ, 709, 611

D’Odorico V., Cristiani S., Romano D., Granato G. L., Danese L., 2004, MNRAS, 351, 976

Elvis M., 2006, Mem. Soc. Astron. Ital., 77, 573

Faucher-Giguère C.-A., Kereš D., 2011, MNRAS, 412, L118

Faucher-Giguère C.-A., Quataert E., Murray N., 2012, MNRAS, 420, 1347

Ferland G. J. et al., 2013, RMxAA, 49, 137

Finn C. W. et al., 2014, MNRAS, 440, 3317

Foltz C. B., Weymann R. J., Peterson B. M., Sun L., Malkan M. A., Chaffee F. H., Jr, 1986, ApJ, 307, 504

Fumagalli M., Prochaska J. X., Kasen D., Dekel A., Ceverino D., Primack J. R., 2011, MNRAS, 418, 1796

Gabel J. R., Arav N., Kim T.-S., 2006, ApJ, 646, 742

Gabel J. R. et al., 2005, ApJ, 631, 741

Ganguly R., Eracleous M., Charlton J. C., Churchill C. W., 1999, AJ, 117, 2594

Gaskell C. M., 1982, ApJ, 263, 79

Hafen Z. et al., 2017, MNRAS, 469, 2292

Hamann F., 1997, ApJS, 109, 279

Hamann F., Barlow T., Cohen R. D., Junkkarinen V., Burbidge E. M., 1997a, in Arav N., Shlosman I., Weymann R. J., eds, ASP Conf. Ser. Vol. 128, Mass Ejection from Active Galactic Nuclei. Astron. Soc. Pac., San Francisco, p. 19

Hamann F., Barlow T. A., Beaver E. A., Burbidge E. M., Cohen R. D., Junkkarinen V., Lyons R., 1995, ApJ, 443, 606

Hamann F., Barlow T. A., Junkkarinen V., 1997a, ApJ, 478, 87

Hamann F., Barlow T. A., Junkkarinen V., Burbidge E. M., 1997b, ApJ, 478, 80

Hamann F., Bristol, 2000, Institute of Physics Publishing, Encyclopedia of Astronomy and Astrophysics, Quasistellar Objects: Intrinsic AGN Absorption Lines, p. 2362

Hamann F., Chartas G., McGraw S., Rodriguez Hidalgo P., Shields J., Capellupo D., Charlton J., Eracleous M., 2013, MNRAS, 435, 133

Hamann F., Ferland G., 1999, ARA\&A, 37, 487

Hamann F., Kanekar N., Prochaska J. X., Murphy M. T., Ellison S., Malec A. L., Milutinovic N., Ubachs W., 2011, MNRAS, 410, 1957

Hamann F., Korista K. T., Ferland G. J., Warner C., Baldwin J., 2002, ApJ, 564,592 
Hamann F., Sabra B., 2004, in Richards G. T., Hall P. B., eds, ASP Conf. Ser. Vol. 311, AGN Physics with the Sloan Digital Sky Survey. Astron. Soc. Pac., San Francisco, p. 203

Hamann F., Tripp T., Rupke D., Veilleux S., 2018, MNRAS, 00, 0

Hamann F. W., Barlow T. A., Chaffee F. C., Foltz C. B., Weymann R. J., 2001, ApJ, 550, 142

Hamann F. W., Netzer H., Shields J. C., 2000, ApJ, 536, 101

Hopkins P. F., Elvis M., 2010, MNRAS, 401, 7

Hopkins P. F., Hernquist L., Cox T. J., Kereš D., 2008, ApJS, 175, 356

Hopkins P. F., Richards G. T., Hernquist L., 2007, ApJ, 654, 731

Hopkins P. F., Torrey P., Faucher-Giguère C.-A., Quataert E., Murray N., 2016, MNRAS, 458, 816

Ho S. H., Martin C. L., Kacprzak G. G., Churchill C. W., 2017, ApJ, 835, 267

Johnson S. D., Chen H.-W., Mulchaey J. S., 2015, MNRAS, 452, 2553

Katz N., Keres D., Dave R., Weinberg D. H., 2003, in Rosenberg J. L., Putman M. E., eds, Astrophysics and Space Science Library, Vol. 281, The IGM/Galaxy Connection. The Distribution of Baryons at $\mathrm{z}=0, \mathrm{p}$. 185

Kauffmann G., Haehnelt M., 2000, MNRAS, 311, 576

Kent S. M., Sargent W. L. W., 1979, ApJ, 230, 667

Kereš D., Katz N., Fardal M., Davé R., Weinberg D. H., 2009, MNRAS, 395,160

Kereš D., Vogelsberger M., Sijacki D., Springel V., Hernquist L., 2012, MNRAS, 425, 2027

King A., 2003, ApJ, 596, L27

Kramida A., Ralchenko Y., Nave G., Reader J., 2018, APS Meeting Abstracts, p. M01.004

Leighly K. M., Dietrich M., Barber S., 2011, ApJ, 728, 94

Martin D. C., Matuszewski M., Morrissey P., Neill J. D., Moore A., Cantalupo S., Prochaska J. X., Chang D., 2015, Nature, 524, 192

Martin D. C., Matuszewski M., Morrissey P., Neill J. D., Moore A., Steidel C. C., Trainor R., 2016, ApJ, 824, L5

McCourt M., Oh S. P., O’Leary R. M., Madigan A.-M., 2018, MNRAS, 473,5407

Misawa T., Charlton J. C., Eracleous M., Ganguly R., Tytler D., Kirkman D., Suzuki N., Lubin D., 2007a, ApJS, 171, 1

Misawa T., Eracleous M., Charlton J. C., Kashikawa N., 2007b, ApJ, 660, 152

Morris S. L., Weymann R. J., Foltz C. B., Turnshek D. A., Shectman S., Price C., Boroson T. A., 1986, ApJ, 310, 40

Murphy T. et al., 2010, MNRAS, 402, 2403

Muzahid S., Srianand R., Arav N., Savage B. D., Narayanan A., 2013, MNRAS, 431, 2885

Nagao T., Marconi A., Maiolino R., 2006, A\&A, 447, 157

Narayanan D., Hamann F., Barlow T., Burbidge E. M., Cohen R. D., Junkkarinen V., Lyons R., 2004, ApJ, 601, 715

Nelson D., Genel S., Vogelsberger M., Springel V., Sijacki D., Torrey P., Hernquist L., 2015, MNRAS, 448, 59

Nestor D., Hamann F., Rodriguez Hidalgo P., 2008, MNRAS, 386, 2055

Netzer H., 1992, in Holt S. S., Neff S. G., Urry C. M., eds, American Institue of Physics Conference Series, 254. Am. Inst. Phys., New York, p. 146

Osterbrock D. E., 1989, Astrophysics of Gaseous Nebulae and Active Galactic Nuclei, University Science Books, Mill Valley, CA, p. 422

Ostriker J. P., Choi E., Ciotti L., Novak G. S., Proga D., 2010, ApJ, 722, 642
Perrotta S. et al., 2016, MNRAS, 462, 3285

Petitjean P., Srianand R., 1999, A\&A, 345, 73

Prochaska J. X., Lau M. W., Hennawi J. F., 2014, ApJ, 796, 140

Rauch M., 1998, ARA\&A, 36, 267

Reeves J. N., Turner M. J. L., 2000, MNRAS, 316, 234

Richards G. T., Vanden Berk D. E., Reichard T. A., Hall P. B., Schneider D. P., SubbaRao M., Thakar A. R., York D. G., 2002, AJ, 124, 1

Richards G. T. et al., 2006, ApJS, 166, 470

Robson E. I., Gear W. K., Smith M. G., Ade P. A. R., Nolt I. G., 1985, MNRAS, 213, 355

Rupke D. S. N., Gültekin K., Veilleux S., 2017, ApJ, 850, 40

Rupke D. S. N., Veilleux S., 2013, ApJ, 768, 75

Sanders D. B., Soifer B. T., Elias J. H., Madore B. F., Matthews K., Neugebauer G., Scoville N. Z., 1988, ApJ, 325, 74

Sargent W. L. W., Boksenberg A., Young P., 1982, ApJ, 252, 54

Scannapieco E., Oh S. P., 2004, ApJ, 608, 62

Schaye J., 2001, ApJ, 559, 507

Schaye J., Carswell R. F., Kim T.-S., 2007, MNRAS, 379, 1169

Schneider D. P. et al., 1993, ApJS, 87, 45

Shen Y. et al., 2007, AJ, 133, 2222

Shen Y. et al., 2016, ApJ, 831, 7

Shull J. M., Stevans M., Danforth C. W., 2012, ApJ, 752, 162

Silk J., Rees M. J., 1998, A\&A, 331, L1

Simon L., 2011, High Redshift Quasar Abundances and Environments: Connecting Black Hole and Host Galaxy Evolution. University of Florida, USA

Simon L. E., Hamann F., 2010, MNRAS, 407, 1826

Simon L. E., Hamann F., Pettini M., 2012, in Chartas G., Hamann F., Leighly K. M., eds, ASP Conf. Series Vol. 460, AGN Winds in Charleston. Astron. Soc. Pac., San Francisco, p. 52

Sramek R. A., Weedman D. W., 1980, ApJ, 238, 435

Srianand R., Petitjean P., 2000, A\&A, 357, 414

Steidel C. C., Sargent W. L. W., 1991, ApJ, 382, 433

Suresh J., Bird S., Vogelsberger M., Genel S., Torrey P., Sijacki D., Springel V., Hernquist L., 2015, MNRAS, 448, 895

Tayal S. S., 2008a, ApJS, 179, 534

Tayal S. S., 2008b, VizieR Online Data Catalog, 348

Tripp T. M., Lu L., Savage B. D., 1996, ApJS, 102, 239

Tytler D., Fan X.-M., 1992, ApJS, 79, 1

Tytler D., O'Meara J. M., Suzuki N., Kirkman D., Lubin D., Orin A., 2004, AJ, 128, 1058

Ulrich M.-H., 1989, A\&A, 220, 71

Veilleux S. et al., 2009, ApJ, 701, 587

Wang H., Wang T., Zhou H., Liu B., Wang J., Yuan W., Dong X., 2011, ApJ, 738,85

Warner C., Hamann F., Dietrich M., 2004, ApJ, 608, 136

Weymann R. J., Morris S. L., Foltz C. B., Hewett P. C., 1991, ApJ, 373, 23

Weymann R. J., Williams R. E., Peterson B. M., Turnshek D. A., 1979, ApJ, 234,33

Wild V. et al., 2008, MNRAS, 388, 227

Wise J. H., Eracleous M., Charlton J. C., Ganguly R., 2004, ApJ, 613, 129

York D. G. et al., 2000, AJ, 120, 1579

This paper has been typeset from a $\mathrm{T}_{\mathrm{E}} \mathrm{X} / \mathrm{L} \mathrm{A} \mathrm{T}_{\mathrm{E}} \mathrm{X}$ file prepared by the author. 